\title{
«La sphère étroite»: Jean-Jacques Rousseau e la costruzione dello spazio
}

\section{Marco Menin}

\section{(2) OpenEdition}

1 Journals

\section{Edizione digitale}

URL: http://journals.openedition.org/studifrancesi/8149

DOI: ERREUR PDO dans/localdata/www-bin/Core/Core/Db/Db.class.php L.34 : SQLSTATE[HY000]

[2006] MySQL server has gone away

ISSN: 2421-5856

\section{Editore}

Rosenberg \& Sellier

\section{Edizione cartacea}

Data di pubblicazione: 1 mai 2009

Paginazione: $34-58$

ISSN: 0039-2944

Notizia bibliografica digitale

Marco Menin, ««La sphère étroite»: Jean-Jacques Rousseau e la costruzione dello spazio», Studi Francesi [Online], 157 (LIII | I) | 2009, online dal 30 novembre 2015, consultato il 07 janvier 2021. URL: http://journals.openedition.org/studifrancesi/8149 ; DOI: https://doi.org/ERREUR PDO dans / localdata/www-bin/Core/Core/Db/Db.class.php L.34 : SQLSTATE[HY000] [2006] MySQL server has gone away

\section{(c) (i) (9)}

Studi Francesi è distribuita con Licenza Creative Commons Attribuzione - Non commerciale - Non opere derivate 4.0 Internazionale. 


\section{«La sphère étroite»: Jean-Jacques Rousseau e la costruzione dello spazio}

J'étois déterminé ... à me renfermer pour le reste de mes jours dans la sphére étroite et paisible pour laquelle je me sentois né.

Jean-Jacques Rousseau, Confessions.

Nel pensiero di Jean-Jacques Rousseau l'insieme delle relazioni che lega l'essere umano all'ambiente circostante implica una continua influenza reciproca tra i due termini: se da una parte lo spazio esterno agisce inevitabilmente sulla costituzione sensibile dell'uomo, dall'altra la personalità individuale può apportare all'oggettiva percezione del mondo esteriore modificazioni profonde e determinanti. Una particolare concezione dello spazio diviene così costitutiva della visione rousseauiana del mondo e la sua intera produzione letteraria si può interpretare come la ricerca inesausta di uno spazio ideale, un luogo privilegiato dove l'individuo possa raggiungere la felicità e realizzarsi pienamente come soggetto morale: un simile spazio dovrà presentare caratteristiche ben precise in grado di rispecchiare la complessità della vita intima del soggetto, sino a condurre a una perfetta coincidenza tra l'interiorità e l'esteriorità.

Il motivo della costruzione dello spazio assume una particolare rilevanza in ogni fase dello sviluppo del pensiero di Rousseau, poiché rappresenta il tentativo più compiuto di una conciliazione tra quelle due esigenze apparentemente contraddittorie che rappresentano i poli dell'universo rousseauiano: retraite e univers, cioè l'autolimitazione e lo slancio espansivo, la costrizione e la dilatazione ${ }^{1}$.

Questa duplicità è riscontrabile nello stesso concetto di spazio, che concerne sia la natura dell'esteriorità in generale, sia la possibilità del rapporto estrinseco con gli oggetti. Sebbene la concezione dello spazio in sé non sia un problema agli occhi di Jean-Jacques - che accetta implicitamente la definizione dello spazio come luogo, cioè come posizione di un corpo tra gli altri corpi - essa si basa su una complessa teoria della sensibilità nella quale si intrecciano inestricabilmente il piano fisico e quello morale. Proprio quest'ultimo aspetto caratterizza in maniera determinante il processo di costruzione spaziale, che non sarà mai un'operazione neutra, ma sempre legata alla ricerca della felicità del soggetto; essa non conduce soltanto alla conoscenza degli oggetti esterni, ma diventa un importante strumento attraverso il quale l'individuo compie la conoscenza di se stesso, sino alla realizzazione di quella coincidenza, tipicamente rousseauiana, tra la quête du bonbeur e la quête de soi.

In quest'ottica è possibile comprendere la ricchezza delle dimensioni spaziali reperibili nell'opera di Rousseau. Tra le sue pagine sono infatti disseminate immagini di grande vivezza relative ai luoghi più differenti: dai primi insediamenti civili del Discours sur l'origine et les fondements de l'inégalité parmi les hommes, alla rievocazione della virtuosa Roma del Contrat Social, dalla comunità di Clarens di Julie ou la Nouvelle Hélö̈se, alla casa-osservatorio in cui Émile compie la propria educazione

(1) Sulla fondamentale coppia oppositiva retraite e univers cfr. P. BuRgELIN, La philosophie de l'existence de J.-J. Rousseau, Paris, P.U.F., 1952, in particolar modo il capitolo Expansion, pp. 49-58; H. Gouhier, Les méditations métaphysiques de
Jean-Jacques Rousseau, Paris, Vrin, 1970 (trad. it. di M. Garin, Filosofia e religione in J.-I. Rousseau, Roma-Bari, Laterza, 1977), in particolar modo il capitolo Expansion et resserrement selon Rousseau, pp. 107-117. 
negativa. Ancora più evidente appare la centralità di questo tema negli scritti autobiografici ${ }^{2}$, vere e proprie gallerie di luoghi che sono cornice di attimi di intensa felicità come di momenti di cupa amarezza: ai luoghi ameni dell'infanzia e della giovinezza, quali Bossey, Chambéry o le Charmettes, tratteggiati con preziosa profondità nelle Confessions, si affiancano gli inquietanti luoghi teatro del nero complotto dei Dialogues, sino a giungere agli incantevoli rifugi nascosti delle Rêveries, ove il promeneur solitaire può abbandonarsi al senso puro dell'esistenza contemplando il lieve moto delle acque o la bellezza di un fiore.

Anche da un punto di vista meramente cronologico, l'intera produzione di Rousseau è significativamente racchiusa tra luoghi che assumono una valenza particolare. Il titolo dei primi versi che egli considerò «l'ouvrage de mon cœur» ${ }^{3}$ è infatti Le verger de Madame la baronne de Warens. Quarant'anni dopo questi versi convenzionali dedicati al frutteto di "Maman" - nei quali tuttavia già emerge la lacerante opposizione tra la pura innocenza campestre e l'opaca torbidezza del mondo - la sua ultima composizione, le Rêveries, si chiuderà nuovamente con la descrizione di un luogo, l'incantato rifugio delle Charmettes.

La concezione rousseauiana dello spazio e il valore simbolico attribuito ai luoghi si sono imposti relativamente tardi all'attenzione della critica; essi sono stati per lo più affrontati nell'ambito di una settorializzazione specifica, piuttosto che secondo una considerazione globale del pensiero di Rousseau. Gli studi biografici, pur dedicando ampio spazio al mondo "spaziale" rousseauiano, si concentrano generalmente - tranne alcuni quadri complessi come quelli di Raymond Trousson o Maurice Cranston ${ }^{4}$ - su aspetti talmente specifici da rivelarsi più interessanti per uno studio dell'uomo Rousseau che per quello del suo pensiero. Ben più utili si rivelano gli studi di critica letteraria, tra i quali si possono rintracciare numerosi contributi sull'estetica del paesaggio di Rousseau e sul valore simbolico che particolari luoghi reali o immaginari assumono nella sua opera. Fondamentale, in tale ambito, è stata l'introduzione nella critica di alcune categorie esistenzialistiche; questa svolta, avvenuta nei primi anni della seconda metà del secolo scorso grazie a interpreti come Pierre Burgelin e Jean Starobinski ${ }^{5}$, ha consentito di superare quelle interpretazioni riduttive che relegavano le tensioni interiori di Jean-Jacques e le sue reazioni all'ambiente esclusivamente nel dominio della malattia. L'introduzione di ipotesi psicoanalitiche nell'analisi letteraria ha fornito nuovi elementi a sostegno della rilevanza dell'immaginazione dello spazio e della sua rappresentazione. Infine, per quel che riguarda studi di carattere filosofico o di storia delle idee in senso stretto - a lungo impigliati in dispute sul carattere "totalitario" o "liberale" del pensiero di Rousseau o su altre controversie ideologiche - l'attenzione si è concentrata per lo più sull'aspetto politico del suo pensiero, privilegiando così un'analisi dello spazio pubblico e collettivo. Nonostante la ricchezza di spunti di riflessione che questi approcci metodologici differenti offrono per un'analisi

(2) Lionello Sozzi ha osservato, riferendosi in maniera specifica agli scritti autobiografici, che «pochi autori ci lasciano un desiderio così struggente di rivisitare i "luoghi ameni", nell'idea confortante pur se illusoria di poter rivivere, in compagnia di JeanJacques, l'incanto anche effimero e sbiadito di quegli istanti di felicità». L. SozzI, Introduzione, in J.-J. Rousseau, Scritti autobiografici, a cura di L. SozzI, Torino, Einaudi-Gallimard, 1997, p. XXI.

(3) J.-J. Rousseau, Avertissement, in Le verger de Madame la baronne de Warens, in Euvres complètes, curatori generali B. GAGNEBIN e M. RAYMOND, Paris, Gallimard («Bibliothèque de la Pléiade»), 1959-1995, 5 voll., vol. II, p. 1123 [in seguito
O.C. seguito dal numero romano del volume].

(4) Cfr. R. Trousson, Jean-Jacques Rousseau, 2 voll.; vol. I, La marche à la gloire; vol. II, Le deuil éclatant du bonbeur, Paris, Tallandier, 1988-1989. Nuova edizione in un unico volume, Jean-Jacques Rousseau, Paris, Tallandier, 2003; M. CRAnSton, Jean-Jacques, 2 voll.; vol. I, The early Life and Works, 1712-1754, vol. II, The noble savage, 1745 1762, London, Allen Lane, 1983-1991.

(5) Cfr., oltre alla già menzionata La philosophie de l'existence de J.-J. Rousseau di P. Burgelin, J. StAROBINSKI, Jean-Jacques Rousseau. La transparence et l'obstacle, Paris, Gallimard, 1957 (trad. it. di R. Albertini, Bologna, il Mulino, 1982). 
più approfondita della concezione rousseauiana dello spazio, non esistono tuttavia studi specifici di ampio respiro sull'argomento, che viene affrontato per lo più in relazione ad altri nodi problematici.

Le pagine che seguono si propongono non soltanto di mostrare la centralità della concezione dello spazio nell'evoluzione del pensiero rousseauiano, ma anche di mettere in luce come vi sia una profonda coerenza nella delineazione del processo di costruzione spaziale il quale, pur colorandosi di varie sfumature che rispecchiano la storia intellettuale dell'autore, sembra riconducibile a un universo di discorso unitario.

Punto di partenza per tale indagine è l'approfondimento della concezione rousseauiana della sensibilità, nella quale sono sviluppate con grande originalità premesse sensistiche mai estinte: se è vero per Rousseau che ogni elemento esterno agisce sulla nostra "macchina" corporea e, di conseguenza, sulla nostra anima, è altrettanto vero (e in questo consiste l'ambizione filosofica più ardita di Jean-Jacques) che è dato a noi governare nella loro origine i sentimenti da cui ci lasciamo dominare. Si tratterà poi di delineare dapprima le caratteristiche dello spazio ideale e, in seguito, di mostrare come tali caratteristiche trovino un'espressione simbolica particolarmente significativa nell'immagine della sfera, vera e propria immaginechiave della spazialità rousseauiana che racchiude in sé tutte le caratteristiche del processo di costruzione spaziale ${ }^{6}$.

L'attribuzione di una particolare rilevanza, sia teorica che letteraria, a un'immagine o a un simbolo è operazione peculiare alla forma mentis rousseauiana, in cui la catarsi razionale affonda le proprie radici nella sensazione e nell'immediata reazione emotiva del soggetto a contatto con il mondo. Rousseau pensatore è innanzitutto, come egli stesso amava definirsi, un homme à sensation, ovvero «un filosofo che sente prima di pensare, e pensa per immagini» ${ }^{7}$. Egli avvertì sempre con particolare forza quella che si potrebbe definire l' "emergenza dell'immagine", cioè la necessità di concretizzare le idee astratte in espressioni simboliche e immagini concrete, le quali finiscono con il costituire la vera e propria impalcatura della sua riflessione filosofica, meno manifesta rispetto ad una costruzione teorica sistematica, ma non necessariamente meno solida.

\section{Alle radici dell'interazione con lo spazio: sensibilità e morale sensitiva}

La relazione con lo spazio esterno caratterizza l'esistenza umana sin dai primi momenti di vita: «Nous naissons sensibles, et dès nôtre naissance nous sommes affectés de diverses maniéres par les objets qui nous environnent. Sitôt que nous avons, pour ainsi dire, la conscience de nos sensations, nous sommes disposés à rechercher ou à fuir les objets qui les produisent» ${ }^{8}$. Tale relazione è destinata a diventare via via più complessa con il trascorrere del tempo: se in un primo momento essa sarà limitata a una meccanica ricerca degli oggetti «selon qu'elles nous sont agréables ou

(6) L'unico studio, a mia conoscenza, che approfondisce in modo specifico la rilevanza dell'immagine della sfera nel pensiero di Rousseau è il capitolo a lui dedicato da G. Poulet in Les métamorphoses du cercle, Paris, Plon, 1961, pp. 102-129 (trad. it. di G. Bogliolo, Milano, Rizzoli, 1971). Poulet, nella convinzione che la pratica letteraria sia un tentativo di presa di possesso della realtà, indaga lo schema del circolo inteso come la figurazione simbolica dello spazio di percezione del reale da parte dell'uomo che si trova nel suo centro. Egli è così più interessato ai vari modi in cui i diversi scrittori, compreso Rousseau, "restituiscono" o "creano" lo spazio, trascurando nella sua analisi le suggestioni sensistiche del pensiero del Ginevrino. Cfr. inoltre il breve paragrafo La «sphère» in G. BESSE, Jean-Jacques Rousseau. L'apprentissage de l'bumanité, Paris, Edition Sociales, 1988, pp. 26-28.

(7) P. CAsinI, Introduzione a Rousseau, Roma-Bari, Laterza, 1974, p. 9.

(8) J.-J. Rousseau, Émile, I, O.C., IV, p. 248. 
déplaisantes», in seguito sarà caratterizzata «selon la convenance ou disconvenance que nous trouvons entre nous et ces objets» e poi «selon les jugemens que nous en portons sur l'idée de bonheur ou de perfection que la raison nous donne»?.

$\grave{E}$ evidente come la relazione con lo spazio esterno non sia meramente passiva, pur basandosi inevitabilmente su quella recettività che pone l'uomo sullo stesso livello di tutti gli altri animali. Egli, infatti, non subisce soltanto l'ambiente, ma reagisce ad esso e «de cette action et réaction viennent les révolutions de la terre et l'agitation continuelle de ses habitans» ${ }^{10}$. Proprio la libera reazione allo stimolo esterno consente di "costruire" lo spazio circostante, modificandolo non solo sulla base di una causalità meccanica, ma anche su quella di istanze morali: al semplice "piacere" si sostituiscono infatti la "compatibilità" e la "felicità" ${ }^{11}$. La stessa coscienza delle proprie sensazioni allontana l'essere umano dalla mera passività, dispiegando innanzi a lui la dimensione morale:

[...] nous sentons avant de conoitre. [...] Les actes de la conscience ne sont pas des jugemens, mais des sentimens; quoique toutes nos idées nous viennent du dehors, les sentimens qui les apprécient sont au dedans de nous, et c'est par eux seuls que nous connoissons la convenance ou disconvenance qui existe entre nous et les choses que nous devons rechercher ou fuir ${ }^{12}$.

La relazione con lo spazio esterno viene così a caratterizzarsi per due prerogative fondamentali: innanzitutto si tratta di un processo che oscilla sempre tra la passività e l'attività, garantendo non solo una continuità, ma anche una reciproca influenza, tra la dimensione fisica (l'animalità dell'uomo) e quella morale (la sua qualità di agente libero $)^{13}$. Inoltre, sarà proprio questo risvolto etico del processo di costruzione spaziale a suscitare l'interesse di Rousseau e a rendere comprensibile il grande valore simbolico che attribuisce ai luoghi. Egli, infatti, considera sempre gli individui «dans leur réalité totale, au sein d'un monde, dans une situation donnée. Cette totalité implique non seulement les conditions géographiques ou physiologiques, les formes sociales, mais aussi l'univers des valeurs, les aspirations morales, esthétiques et religieuses où se révèlent les structures ontologiques du monde et de l'homme, enfin la complexité infinie des personnalités absolument libres» ${ }^{14}$. Il dato esclusivamente sensibile e fisiologico assume rilevanza in quanto condizione indispensabile per spiegare quello morale.

Una conferma determinante dell'esistenza di un nesso tra la capacità dell'essere umano di interagire in modo via via più raffinato con l'ambiente circostante e il suo sviluppo morale è offerta dal fatto che l'importanza dei luoghi è direttamente proporzionale all'avanzare del processo d'incivilimento. Nello stato di natura l'uomo si trova in una condizione di assoluta indifferenziazione, che rende impossibile l'attribuzione di un qualsiasi significato allo spazio esterno: egli, infatti, non è «attacché à aucun

(9) Ibidem.

(10) J.-J. Rousseau, Essai sur l'origine des langues, O.C., V, p. 394. La coppia concettuale di "azione e reazione", ricavata dagli assiomi della meccanica razionale, è usata da Jean Starobinski come ipotesi di lettura del linguaggio politico, ideologico e letterario settecentesco. Cfr. J. STAROBINSKI, Action et reaction. Vie et aventures d'un couple, Paris, Seuil, 1999 (trad. it. di C. Colangelo, Torino, Einaudi, 2001).

(11) L'agrément, la convenance e il bonheur si possono considerare tre tappe fondamentali dello sviluppo umano: «Jusqu'ici nous n'avons connu de loi que celle de la necessité: maintenant nous avons égard à ce qui est utile; nous arriverons bientôt à ce qui est convenable et bon». J.-J. RoussEAu, Émile, III, O.C., IV, p. 429.

(12) Ivi, IV, O.C., IV, p. 599.

(13) Il fine naturale dell'essere umano è un fine morale, cioè il bonbeur: «Il faut être heureux, cher Emile; c'est la fin de tout être sensible; c'est le prémier desir que nous imprima la nature, et le seul qui ne nous quite jamais». Ivi, V, O.C., IV, p. 814.

(14) P. Burgelin, op. cit., p. 29. 
lieu» ${ }^{15}$. Lo spazio rimane in tale situazione originaria intrinsecamente racchiuso in una dimensione di assoluta staticità: «Le spectacle de la Nature lui devient indifférent, à force de lui devenir familier. C'est toujours le même ordre, ce sont toujours les mêmes révolutions» ${ }^{16}$. L'uomo naturale subisce così passivamente l'azione degli oggetti esterni: «Appercevoir et sentir sera son premier état, qui lui sera commun avec tous les animaux $\rangle^{17}$. Tuttavia, proprio la capacità di reagire a quelli che sono gli impulsi provenienti dall'esterno, capacità non innata ma acquisita grazie all'intervento di quella facoltà esclusivamente umana che è la perfectibilité, segna il vero discrimine (più ancora dell'intelligenza, nel cui caso la differenza è quantitativa e non qualitativa) tra l'essere umano e quella "macchina ingegnosa" che è l'animale: «La Nature commande à tout animal, et la Bête obéït. L'homme éprouve la même impression, mais il se recconnoît libre d'acquiescer, ou de resister; et c'est surtout dans la conscience de cette liberté que se montre la spiritualité de son ame» ${ }^{18}$.

Il processo di civilizzazione si può così interpretare come la crescente capacità di agire attivamente nei confronti dell'ambiente esterno: non a caso esso è significativamente racchiuso - nell'analisi condotta nel Discours sur l'origine de l'inégalité - entro due luoghi dalla grande portata simbolica: le capanne delle prime associazioni umane e il terreno cintato dal «vrai fondateur de la société civile» ${ }^{19}$, primo esempio di "luogo" inteso come nozione culturalmente determinata. La relazione con lo spazio si caratterizza così per una connotazione morale, che rende irriducibile la complessità dell'essere umano - sia per quel che riguarda la ricchezza della vita interiore, sia per quel che riguarda la relazione con gli oggetti esterni - all'applicazione di leggi esclusivamente fisiche e meccaniche.

Per comprendere l'originalità e l'audacia speculativa di questa teoria è necessario analizzare più da vicino la nozione di "sensibilité", vera e propria chiave di volta dell'antropologia rousseauiana. Questa facoltà, che accomuna per altro l'bomme de la nature all'bomme de l'bomme, ha un'assoluta preminenza nella formazione dell'essere umano. Infatti, «exister pour nous, c'est sentir; notre sensibilité est incontestablement antérieure à nôtre intelligence, et nous avons eu des sentimens avant des idées. Quelle que soit la cause de nôtre être, elle a pourvû à nôtre conservation en nous donnant des sentimens convenables à nôtre nature, et l'on ne sauroit nier qu'au moins ceux-là ne soient innés $\rangle^{20}$. Questa priorità della sensazione sarà sempre ribadita, e addirittura accentuata, anche a livello autobiografico: «Je sentis avant de penser; c'est le sort commun de l'humanité. Je l'eprouvai plus qu'un autre. [...] Je n'avois rien conçu; j'avois tout senti ${ }^{21}$. In breve, per quel che riguarda la sua prerogativa originaria, che è e deve rimanere fondamentale, l'essere umano è eminentemente ricettivo: questo spiega l'influenza dei luoghi e dell'ambiente circostante su di lui. Tuttavia, in Rousseau, «pâtir, c'est subir, mais c'est aussi réagir» ${ }^{22}$; l'uomo può rispondere con un'azione appropriata alle sollecitazioni esterne e le sue passioni non saranno che la risposta all'azione di un oggetto determinato. Una simile posizione non solo garantisce l'interazione dell'uomo con l'ambiente circostante, ma introduce la possibilità

(15) J.-J. Rousseau, Émile, II, O.C., IV, p. 360.

(16) J.-J. Rousseau, Discours sur l'origine de l'inégalité, O.C., III, p. 144.

(17) Ivi, O.C., III, p. 143.

(18) Ivi, O.C., III, pp. 141-142.

(19) Ivi, O.C., III, p. 164.

(20) J.-J. Rousseau, Émile, IV, O.C., IV, p. 600. La preminenza dei sensi nello sviluppo dell'essere umano era già stata teorizzata chiaramente in precedenza: «Les prémiéres facultés qui se forment et se perfectionnent en nous sont les sens. Ce sont donc les prémiéres qu'il faudroit cultiver; ce sont les seules qu'on oublie ou celles qu'on néglige le plus. Exercer les sens n'est pas seulement en faire usage, c'est apprendre à bien juger par eux, c'est apprendre, pour ainsi dire, à sentir». Ivi, II, O.C., IV, p. 380.

(21) J.-J. Rousseau, Confessions, I, O.C., I, p. 8.

(22) E. Gilson, La méthode de M. de Wolmar, in Les idées et les lettres, Paris, Vrin, 1955, p. 280. 
di trovare un perfetto equilibrio tra le due dimensioni. In quello spazio ideale a cui ambisce inesaustamente l'animo rousseauiano ci sarà una perfetta armonia tra l'essere umano e il luogo circostante, tanto che, venendo meno la necessità di qualsiasi reazione, potranno esservi un'immedesimazione e una fusione assolute. Proprio tale aspetto caratterizza gli abitanti del mondo ideale:

Figurez-vous donc un monde idéal semblable au nôtre, et neanmoins tout différent. La nature y est la même que sur notre terre, mais l'economie en est plus sensible, l'ordre en est plus marqué, le spectacle plus admirable; les formes sont plus élégantes, les couleurs plus vives, les odeurs plus suaves, tous les objets plus interessans. Toute la nature y est si belle que sa contemplation enflammant les ames d'amour pour un si touchant tableau leur inspire avec le desir de concourir à ce beau système la crainte d'en troubler l'harmonie, et delà nait une exquise sensibilité qui donne à ceux qui en sont doués des jouissances immédiates, inconnues aux cœurs que les mêmes contemplations n'ont point avivés ${ }^{23}$.

Già da questi pochi accenni ci si rende conto di come la sensibilité si ritrovi investita di funzioni multiple e debba essere considerata attentamente: «Tâchons de commencer par bien entendre ce mot de sensibilité, auquel, faute de notions exactes, on applique à chaque instant des idées si vagues et souvent si contradictoires» ${ }^{24}$. Bisogna infatti distinguere, come viene fatto con chiarezza nei Dialogues, da un lato «une sensibilité physique et organique, qui, purement passive, paroit n'avoir pour fin que la conservation de notre corps et celle de notre espéce par les directions du plaisir et de la douleur» e dall'altro una «sensibilité [...] active et morale qui n'est autre chose que la faculté d'attacher nos affections à des êtres qui nous sont étrangers» ${ }^{25}$.

La sensibilità fisica non sarà altro che la sensation, cioè quel sostrato che accomuna non solo l'bomme de la nature all'bomme de l'bomme, ma anche l'essere umano a tutti gli altri animali. Essa riguarda, così, il livello meramente corporale: i colori, i suoni, gli odori, l'azione che esercitano gli elementi, il rumore o il silenzio; tutto questo agisce sull'essere umano e lo riempie di sensazioni nuove. Saranno così tali sensazioni a scatenare desideri e passioni, le quali a loro volta acuiranno le facoltà intellettive che reagiranno in senso inverso su quest'ultime. Nell'Émile, in cui l'intero processo educativo sarà costruito proprio su un corretto sviluppo della sensibilità, si arriverà addirittura a ipotizzare una "raison sensitive" anteriore a quella intellettuale e che costituirebbe il fondamento di quest'ultima:

Les prémiers mouvemens naturels de l'homme étant donc de se mesurer avec tout ce qui l'environne et d'éprouver dans chaque objet qu'il apperçoit toutes les qualités sensibles qui peuvent se rapporter à lui, sa prémiére étude est une sorte de physique expérimentale rélative à sa propre conservation. [...] Tandis que ses organes délicates et fléxibles peuvent s'ajuster aux corps sur lesquels ils doivent agir, tandis que ses sens encore purs sont éxempts d'illusions, c'est le tems d'éxercer les uns et les autres aux fonctions qui leur sont propres, c'est le tems d'apprendre à connoitre les rapports sensibles que les choses ont avec nous. Comme tout ce

(23) J.-J. Rousseau, Dialogues, I, O.C., I, p. 668.

(24) Ivi, II, O.C., I, p. 805.

(25) Ibidem. Rispetto ai materialisti, Rousseau salvaguarda la distinzione tra sensibilità passiva, a livello sensoriale, e sensibilità attiva, a livello intellettuale. Lo spirito è indipendente dalla sensibilità e dalla materia, benché da essa tragga i suoi dati. Helvétius, in De l'Esprit, spiega le funzioni dello spirito esclusivamente attraverso la sensibilità fisica. Sull'esemplare dell'opera donatogli dallo stesso Helvétius nel 1758, Rousseau scrisse delle note marginali riportate da Pierre-Maurice Masson: «Il me semble qu'il faudroit distinguer les impressions purement organiques et locales des impressions universelles qui affectent tout l'individu. Les prémiéres ne sont que de simples sensations, les autres sont des sentimens». J.-J. Rousseau, Notes sur "De l'Esprit", O.C., IV, p. 1121. A tale proposito cfr. P.M. Masson, Rousseau contre Helvétius, in «Revue d'histoire littéraire de la France», XVIII, 1, 1911, pp. 103-124. 
qui entre dans l'entendement humain y vient par les sens, la prémiére raison de l'homme est une raison sensitive ${ }^{26}$.

Queste riflessioni conducono inevitabilmente a estendere quello che è il ruolo tradizionale della sensibilità fisica: essa ha infatti la funzione fondamentale di annunciare all'uomo non solo l'esistenza del mondo esteriore, ma anche la propria, poiché la graduale coscienza che l'uomo prende di se stesso (grazie all'azione della perfettibilità) deve passare necessariamente per l'ambiente esterno ${ }^{27}$. La sensibilità finisce così con il diventare costitutiva dell'io ${ }^{28}$. Questo concetto è stato messo in rilievo da Jean Starobinski: «"Qui suis-je?". La réponse à cette question est instantanée. "Je sens mon cœur". Tel est le privilège de la connaissance intuitive, qui est présence immédiate à soi-même, et qui se constitue tout entière dans un acte unique du sentiment. Pour Jean-Jacques, la connaissance de soi n'est pas un problème: c'est une donnée» ${ }^{29}$.

$\mathrm{Ci}$ si trova qui dinanzi a una vera e propria inversione di valori rispetto alla costruzione psicologica della filosofia tradizionale; tale inversione è stata espressa in maniera particolarmente efficace da colui che si può considerare il più importante "discepolo" di Rousseau, Jacques-Henri-Bernardin de Saint-Pierre: «Je substitue donc à l'argument de Descartes celui-ci, qui me paraît et plus simple et plus général: je sens, donc j'existe. Il s'étend à toutes nos sensations physiques, qui nous avertissent bien plus fréquemment de notre existence que la pensée» ${ }^{30}$. Questa nuova relazione tra sensibilità e ragione costituisce quella che Etienne Gilson ha definito «une révolution profonde dans la conception classique de l'homme» ${ }^{31}$. Per la prima volta, infatti, sarebbe ribaltata sistematicamente quella visione che affida la condotta della vita umana alla ragione e ne fa il fondamento dell'umanità stessa. Una tale visione era stata centrale nella filosofia occidentale sin da Platone, che l'aveva rappresentata attraverso la celebre immagine della biga alata del Fedro ${ }^{32}$. Secondo questa suggestiva metafora ripresa da Gilson,

Rousseau est le premier qui ait concédé aux chevaux le droit de conduire le cocher et fait un devoir au cocher de se laisser conduire. Car le cocher n'est pas seulement inutile, il est nuisi370 .

(26) J.-J. Rousseau, Émile, II, O.C., IV, pp. 369-

(27) A tale proposito, bisogna rilevare come anche Diderot, a lungo amico fraterno di Jean-Jacques, avesse sviluppato una posizione analoga: «Les sensations font sortir l'ame hors d'elle-même, en lui donnant l'idée confuse d'une cause extérieure qui agit sur elle, parce que les sensations sont des perceptions involontaires. [...] Nos sensations font la preuve la plus convaincante que nous ayons de l'existence de la matiere. C'est par elles que Dieu nous avertit de notre existence». D. DIDEROT, voce «Sensations», Encyclopédie, in Euvres complètes, a cura di J. AsSÉZAT e M. TOURneuX, Paris, 1875 sgg., voll. 20, vol. XVII, Encyclopédie. Voyages. Euvres diverses, p. 119. Limportanza della relazione con l'ambiente esterno, come ha mostrato Robert Mauzi, era d'altronde una prerogativa centrale per tutto il pensiero contemporaneo: «Exister pour l'homme du XVIII' siècle, c'est d'abord être en relation, se sentir solidement rivé aux choses et façonné par elles, gorgé d'elles. Aussi trouvera-t-il la preuve et le contenu même de son existence dans les sensations». R. MAUZI, L'idée de bonbeur dans la littérature et la pensée française au XVIII' siècle, Paris, Colin, 1960, p. 314.
(28) Basti richiamare, a tale proposito, un celebre passo posto in apertura della Profession de foi $d u$ Vicaire savoyard: «J'existe et $\mathrm{j}$ 'ai des sens par lesquels je suis affecté. Voilà la prémiére vérité qui me frape, et à laquelle je suis forcé d'acquiescer. Ai-je un sentiment propre de mon existence, ou ne la sens-je que par mes sensations? Voilà mon prémier doute, qu'il m'est, quant à présent, impossible de résoudre. Car étant continuellement affecté de sensations, ou immédiatement, ou par la mémoire, comment puis-je savoir si le sentiment du moi est quelque chose hors de ces mêmes sensations, et s'il peut être indépendant d'elles?». J.-J. RoussEAu, Emile, IV, O.C., IV, pp. 570-571.

(29) J. STAROBINSKI, Jean-Jacques Rousseau. La transparence et l'obstacle, cit., p. 216.

(30) B. De Saint-Pierre, Etudes de la Nature, XII, in Euvres complètes de Jacques-Henri-Bernardin de Saint-Pierre, mises en ordre et précédées de la vie de l'auteur, a cura di L. Aimé-Martin, Paris, Mequignon-Marvis, 1818 (riproduzione anastatica su archivio elettronico), 12 voll., vol. V, p. 10.

(31) E. GILSON, op. cit., p. 277.

(32) Cfr. Platone, Fedro, 246 a e ss. 
ble. C'est par sa faute qu'arrivent tous les accidents de voiture et les chevaux iraient bien mieux tout seuls. Il a donc laissé le cocher à sa place, mais en lui recommandant de ne tenir les rênes que pour se laisser tirer [...].Voilà quelle est l'essence du rousseauisme de Rousseau, et par là-même celle de tous les rousseauismes: pédagogiques, politiques, métaphysiques, littéraires et musicaux. Son triomphe fut instantané, parce qu'il libérait dans l'homme l'animal qui n'est qu'une partie de l'homme. Bref, le rousseauisme, c'est la démission de la raison ${ }^{33}$.

Da queste riflessioni risulta ormai evidente come la sensibilità, accanto a una funzione gnoseologica e, per così dire, metafisica (nel limite in cui è costitutiva dell'io) rivestirà anche una funzione morale, perché sarà alla base di quella ricerca del bonheur che è l'obiettivo ultimo dell'essere umano. Non si tratterà più in questo caso di una "sensibilité physique" passiva, ma di quella "sensibilité active et morale" chiamata anche "sentiment". Questo termine indica ciò che è propriamente umano nella sensibilità ed è inevitabilmente legato al processo di perfettibilità ${ }^{34}$. Si tratterà così di una facoltà ambivalente, perché i sentimenti saranno naturali nella misura in cui riguardano l'istinto e la bontà originaria; tuttavia potranno anche, sotto l'effetto della rivalità sociale $e^{35}$ e lusingati ad esempio dall'amour propre, svilupparsi in tutt'altra direzione $^{36}$. Rousseau distingue infatti tra due forme di amor proprio: l'amor proprio in senso lato, che chiama anche amore di sé (amour de soi), è un sentimento assoluto, naturale e buono per definizione perché assicura l'autoconservazione dell'individuo ed esprime il suo diritto alla vita; l'amor proprio relativo (amour propre) è invece sempre negativo, perché, nascendo dal confronto con gli altri, si configura come sentimento sociale ed è quindi subordinato all'opinione. Proprio a causa di questa duplicità della sensibilità attiva, sarà particolarmente importante studiare e ribadire il legame che essa intrattiene con la moralità, come del resto viene spesso sottolineato nelle pagine rousseauiane:

Tant que sa sensibilité reste bornée à son individu il n'y a rien de moral dans ses actions; ce n'est que quand elle commence à s'étendre hors de lui qu'il prend d'abord les sentimens et ensuite les notions du bien et du mal qui le constituent véritablement homme et partie intégrante de son espéce ${ }^{37}$.

(33) E. GILsOn, op : cit., p. 278. Una simile posizione non implica evidentemente una svalutazione della ragione, ma una sua diversa considerazione. Come ha osservato Pierre Burgelin, «La raison a un contenu et sa voix est celle même de la nature. Pas de raison sans un appel au sensibile, parce qu'il n'y a pas de raison indépendante des sentiments, du corps, ni du monde». P. Burgelin, op. cit., p. 364. Un tentativo di mettere in luce l'insufficienza della ragione per spiegare la totalità del pensiero rousseauiano è rappresentato da M. QVORTRUP, The political philosophy of Jean-Jacques Rousseau: the impossibility of reason, Manchester, Manchester U.P., 2004.

(34) Cfr. J. SGARD, voce «Sentiment», in Dictionnaire de Jean-Jacques Rousseau, a cura di R. TROusSON e F. Eigeldinger, Paris, Champion, 1996, 2 ${ }^{\mathrm{a}}$ ed. 2001, p. 854. Per un'analisi dettagliata di tale tematica si consulti M. GILOT e J. SGARD, Le Vocabulaire du sentiment dans l'auvre de Jean-Jacques Rousseau, Genève-Paris, Slatkine, 1980.

(35) La distinzione tra amour de soi e amour propre è sviluppata in particolar modo nella nota XV del Discours sur l'origine de l'inégalité, in J.-J. RousSEAU, O.C., III, pp. 219-220. Cfr. Y. SaKaKURA, Es- sai sur "l'amour-propre" chez Jean-Jacques Rousseau, in «Etudes Jean-Jacques Rousseau», VII, 1995, pp. 223-239.

(36) La sensibilità attiva, infatti, non ha un valore esclusivamente positivo: «La sensibilité positive dérive immediatement de l'amour de soi. Il est très naturel que celui qui s'aime cherche à étendre son être et ses jouissances, et à s'approprier par l'attachement ce qu'il sent devoir être un bien pour lui. [...] Mais sitot que cet amour absolu dégénere en amour-propre et comparatif, il produit la sensibilité négative; parce qu'aussitot qu'on prend l'habitude de se mesurer avec d'autres, et de se transporter hors de soi pour s'assigner la prémiére et meilleure place, if est impossible de ne pas prendre en aversion [...] tout ce qui étant quelque chose nous empêche d'être tout». J.-J. RousseAu, Dialogues, II, O.C., I, pp. 805-806. Tale concezione ambivalente della sensibilità pare esemplificata, nella Nouvelle Héloïse, dalle ultime parole pronunciate da Julie ormai morente: «On m'a fait boire jusqu'à la lie la coupe amere et douce de la sensibilité». J.-J. RousSEAU, Julie ou la Nouvelle Hélö̈se (VI, 11), O.C., II, p. 733.

(37) J.-J. Rousseau, Émile, IV, O.C., IV, p. 501. 
L'homme est modifié par ses sens, personne n'en doute; mais faute de distinguer les modifications nous en confondons les causes; nous donnons trop et trop peu d'empire aux sensations; nous ne voyons pas que souvent elles ne nous affectent point seulement comme sensations mais comme signes ou images, et que leurs effets moraux ont aussi des causes morales.

[...] nos plus vives sensations agissent souvent par des impressions morales. [...] On apperçoit quelque chose de cet effet moral jusques dans les animaux ${ }^{38}$.

Queste citazioni sono utili per comprendere la relazione che sussiste tra la sensibilité physique, o sensazione, e la sensibilité morale, o sentimento. Innanzitutto, è importante tenere separati questi due aspetti della sensibilità, in quanto il primo unisce gli esseri umani agli altri animali e caratterizza l'esistenza dell'uomo naturale ${ }^{39}$, mentre il secondo è il segno distintivo della società civile ed è contraddistinto da quella duplicità tipica del progresso umano. Inoltre, bisogna sottolineare come non si tratti affatto di due livelli separati, a tal punto che non solo non viene scartata l'idea di un'analogia di funzionamento, ma risulta teorizzata chiaramente persino un'influenza reciproca. «Jean-Jacques», preciserà lo stesso Rousseau nel secondo dei Dialogues, «m'a paru doué de la sensibilité physique à un assez haut degré. Il dépend beaucoup de ses sens et il en dépendroit bien davantage si la sensibilité morale n'y faisoit souvent diversion; et c'est même encor souvent par celle-ci que l'autre l'affecte si vivement $\gg^{40}$. Esiste così una relazione tra la sensibilità fisica e quella morale (e dunque tra l'anima e il corpo), che l'essere umano può sfruttare a proprio vantaggio. Infatti, se la morale - almeno per quel che riguarda questo suo aspetto sensitivo - non è altro che «le bon usage de nos forces dans nos rapports avec le monde» ${ }^{41}$ è ipotizzabile che, creando delle condizioni esterne favorevoli, l'uomo possa migliorare le proprie disposizioni interiori ${ }^{42}$. Se si prova a indagare, all'interno delle pagine rousseauiane, in che cosa possano effettivamente consistere tali condizioni esterne nei confronti delle quali l'uomo sarà sempre in una condizione di recettività e passività, ci si renderà conto di come si tratti essenzialmente di luoghi, e in particolar modo di quegli spazi caratterizzati dall'innocenza naturale, ma ormai culturalmente determinata, che rappresentano il termine ultimo della concezione rousseauiana, più mitica che storica, del progresso.

Una simile interpretazione sembra trovare una significativa conferma nel fatto che uno dei grandi progetti filosofici a cui Rousseau aspirò per tutta la vita, pur senza portarlo mai a compimento, era la stesura di un'opera tesa a indagare proprio il fondamentale rapporto tra sensibilità e morale: egli voleva sapere perché ci si comporta istintivamente in una certa maniera, cercando così di costruire una sorta di morale

(38) J.-J. Rousseau, Essai sur l'origine des langues, XV, O.C., V, pp. 412 e 417.

(39) L'uomo primitivo è in qualche modo esclusivamente la sua sensazione, in quanto «animal borné d'abord aux pures sensations». J.-J. RousseAu, Discours sur l'origine de l'inégalité, O.C., III, p. 164. Già a questo livello, tuttavia, la sensibilità è costitutiva del sentimento dell'esistenza, come ha mostrato Georges Poulet: «Enfin, dans cette sensation pure qui est en même temps pure activité et sentiment de l'existence, l'homme possède le bonheur parfait. Il n'y a aucun contrariété en lui: son moi emplit l'univers, et l'univers emplit son moi. Il vit en lui-même, mais inversement la nature vit en lui». G. POULET, Études sur le temps bumain, Paris, Plon, 1952, p. 163. 807.
(41) P. Burgelin, op. cit., p. 357

(42) Proprio questa complessità pare distinguere la posizione di Rousseau da quella di tutti gli altri autori che esaltarono il "sentiment de la nature". Questi ultimi, come ha sottolineato Robert Mauzi, si fermavano essenzialmente al livello di una sensibilità fisica e passiva: «L'influence des sensations sur l'âme constitue la clé du "sentiment" de la nature au XVIII ${ }^{e}$ siècle, qui n'est pas une exaltation gratuite, un pur élan de la sensibilité, mais une expression particulière du sentiment de l'existence. Il s'élabore presque toujours à partir d'impressions pbysiques, et la nuance affective dont il se colore dépend de la disposition matérielle des objets, de la manière dont les sensations se transmettent à l'âme». Cfr. R. MAuZI, op. cit., pp. 319-320. 
sensitiva. Etienne Gilson e Pierre Burgelin sono stati tra i primi ad attirare l'attenzione su «un projet non exécuté de Rousseau, qu'il expose dans les Confessions. Il s'agit d'un livre conçu en même temps qu'Émile et les Institutions politiques, intitulé la Morale sensitive ou le Matérialisme du sage» ${ }^{43}$. Quest'opera rappresenta uno degli enigmi di più difficile risoluzione per la critica rousseauiana, poiché si tratta di un testo fantasma, di cui non esiste nessun manoscritto né nessun abbozzo preparatorio ${ }^{44}$. Gli unici dati sicuri intorno a questo scritto sono il titolo e il sottotitolo che vengono comunicati all'inizio del nono libro delle Confessions, prima di un riassunto programmatico dello stesso lavoro che merita di essere riportato nella sua interezza:

En sondant en moi-même et en recherchant dans les autres à quoi tenoient ces diverses manieres d'être je trouvai qu'elles dépendoient en grande partie de l'impression antérieure des objets exterieurs, et que modifiés continuellement par nos sens et par nos organes, nous portions sans nous en appercevoir, dans nos idées, dans nos sentimens, dans nos actions mêmes l'effet de ces modifications. Les frapantes et nombreuses observations que $\mathrm{j}$ 'avois recueillies étoient au dessus de toute dispute, et par leurs principes physiques, elles me paroissoient propres à fournir un régime extérieur qui varié selon les circonstances pouvoit mettre ou maintenir l'ame dans l'état le plus favorable à la vertu. Que d'écarts on sauveroit à la raison, que de vices on empêcheroit de naitre si l'on savoit forcer l'économie animale à favoriser l'ordre moral qu'elle trouble si souvent! Les climats, les saisons, les sons, les couleurs, l'obscurité, la lumiére, les élemens, les alimens, le bruit, le silence, le mouvement, le repos, tout agit sur notre machine et sur notre ame par consequent; tout nous offre mille prises presque assurées pour gouverner dans leur origine les sentimens dont nous nous laissons dominer ${ }^{45}$.

La Morale sensitive si doveva probabilmente situare alla confluenza tra una metafisica sensualistica ispirata a Locke e a Condillac e un ideale di saggezza ereditato dai filosofi dell'antichità greco-romana, in particolar modo dagli stoici ${ }^{46}$. Si doveva trattare dunque di un'opera che poneva un rilievo particolare sull'importanza dell'oggetto esterno e, stando al testo rousseauiano, questo oggetto pare legato essenzialmente allo spazio esterno (come conferma il riferimento ai climi, alle stagioni, ai suoni e ai colori) piuttosto che a un'altra persona. Ancora una volta, così, ci si trova dinanzi alla fondamentale differenza tra l'irrilevanza del luogo nello stato di natura stricto sensu e il ruolo centrale che esso gioca nella società civile. Questo scarto è stato messo in luce da Pierre Burgelin: «Un certain état de l'âme en rapport avec une certaine situation: l'instinct animal obtient l'accord automatiquement, notre condition actuelle exige davantage. Si l'homme de la nature se suffit, dans notre nouvelle manière d'exister, "tout ce qu'il y a de moral en moi-même a toujours ses relations hors de moi" $\gg$ ". A partire da queste premesse, lo stesso Burgelin pone in evidenza come il progetto di un "materialismo del saggio" si possa ritrovare nelle altre opere rousseauiane: «La morale sensitive, c'est l'Émile pour la théorie, Julie pour la pratique. Et la loi de l'âme ne saurait s'élever contre la loi du corps sans avoir essayé de s'insinuer en elle» ${ }^{48}$. Prima di lui, Etienne Gilson aveva ipotizzato di considerare proprio un luogo, cioè l'Eliso, la realizzazione paesaggistica di una morale sensitiva organizzata da quel saggio materialista che è Wolmar. Una tale interpretazione conferma, per altro, l'inscindibilità

(43) P. Burgelin, op. cit., p. 356.

(44) Cfr. P. Hartmann, voce «Morale sensitive», in Dictionnaire de Jean-Jacques Rousseau, cit., p. 624.

(45) J.-J. Rousseau, Confessions, IX, O.C., I, p. 409.

(46) Seneca fu il filosofo stoico che influenzò maggiormente Rousseau, il quale tradusse addirit- tura una sua opera (Traduction de l'Apocolokintosis de Sénèque, O.C., V, pp. 1213-1226). Cfr. G. PIRE, De l'influence de Sénèque sur les théories pédagogiques de J.-J. Rousseau, in «Annales Jean-Jacques Rousseau», XXXIII, 1953-55, pp. 57-92.

(47) P. Burgelin, op. cit., p. 363.

(48) Ivi, pp. 361-362. 
del pensiero filosofico di Rousseau da quello letterario e pone in luce come il primo abbia spesso bisogno del secondo per strutturarsi ed esprimersi:

Ce qu'il y a au fond de toute cette histoire, c'est une application romanesque de la Morale sensitive ou le Matérialisme du sage, et en même temps que nous éclairons le roman de Rousseau par sa philosophie, nous tirons du roman quelques lumières sur ce qu'eût été ce traité philosophique dont le plan même a disparu ${ }^{49}$.

Al di là delle inevitabili incertezze sugli argomenti che avrebbero dovuto essere affrontati in quest'opera ${ }^{50}$, sembra lecito trarre almeno due conclusioni con un buon margine di certezza. Innanzitutto, tanto la testimonianza delle Confessions quanto le interpretazioni degli studiosi confermano come i luoghi rivestano un'importante funzione filosofica e simbolica all'interno del pensiero rousseauiano, tanto da risultare inscindibili da alcuni concetti chiave della sua antropologia (cultura e sensibilità); inoltre la volontà di studiare a fondo l'ambiente circostante in relazione con l'individuo non rimane un tentativo isolato o viene lasciato cadere per mancanza di interesse, ma rappresenta una delle linee guida dell'intera produzione di Rousseau, tanto da trovarne ancora menzione in una delle testimonianze più tarde, la ventisettesima carta da gioco:

1. Connois toi toi même

2. Froides et tristes reveries

3. Morale sensitive

Comment dois je me conduire avec mes contemporains

Du mensonge

Trop peu de santé

Eternité des peines

Morale sensitive $e^{51}$.

Non si può così che concordare con Bernard Gagnebin e Marcel Raymond, quando rilevano come «avec la mention "Morale sensitive" [...] se fait jour une préoccupation intellectuelle qui est de tous les instants de la vie de Rousseau, [...] parce qu'elle est en relation étroite avec son expérience et avec son désir de trouver une médication psychologique efficace $»^{52}$. Una posizione simile è sostenuta da Pierre Hartmann: «Écrit fantôme, La Morale sensitive l'est donc encore au sens où il hante secrètement l'œuvre entière de son auteur» ${ }^{53}$.

Questo presunto scritto sul materialismo del saggio, albero esile e forse inesistente, nasconde così dietro di sé una foresta di riferimenti ben più reali che rendono lecito interrogarsi non solo sulla possibilità dell'influenza che il luogo ha sull'individuo ma, addirittura, sulla possibilità di costruire un luogo ideale, dove l'essere umano possa ritrovare, nella piena armonia tra la dimensione fisica e quella morale, la propria dimensione originaria perduta.

(49) E. Gilson, op. cit., p. 293. Anche Robert Mauzi vede nella descrizione di un luogo, in qusto caso l'isola di Saint-Pierre, il momento più significativo per comprendere la teoria rousseauiana della morale sensitiva. Cfr. R. Mauzi, op. cit., pp. 296-299.

(50) Un'ipotetica ricostruzione è tentata nel breve saggio di A. Bruno, Jean-Jacques Rousseau. La morale sensitiva o il materialismo del saggio, Milano, Angeli, 1997.

(51) J.-J. Rousseau, Ébauches des Rêveries, O.C., I, p. 1172 .

(52) B. Gagnebin e M. Raymond, Notes et variantes, in J.-J. Rousseau, O.C., I, p. 1863.

(53) P. Hartmann, voce «Morale sensitive», in Dictionnaire de Jean-Jacques Rousseau, cit., p. 624. 


\section{Lo spazio ideale}

Rousseau fu sempre perfettamente consapevole dell'influenza reciproca tra l'individuo e l'ambiente circostante. Se quest'ultimo agisce inevitabilmente sulla costituzione sensibile, e dunque eminentemente recettiva, dell'essere umano, sono riconosciute allo stesso modo le profonde modificazioni che la personalità individuale può apportare all'oggettiva percezione del mondo esteriore: «Je ne Sais voir qu'autant que je Suis ému; les objets indifférens Sont nuls à mes yeux. [...] Nos rélations Se rapportent toujours plus à nous qu'aux choses $\rangle^{54}$. La complessità della vita interiore non può che complicare l'interpretazione dell'immaginazione rousseauiana dello spazio esterno, tanto più che Jean-Jacques erge se stesso a modello universale dell'essere umano. Negli scritti autobiografici, egli parla spesso dei suoi bruschi cambiamenti di umore, delle rivoluzioni della sua personalità e delle bizzarre oscillazioni del suo carattere, che non gli hanno mai permesso di raggiungere una tanto agognata pace interiore: «Je crois avoir déja remarqué qu'il y a des tems où je suis si peu semblable à moi-même qu'on me prendroit pour un autre homme de caractére tout opposé» ${ }^{55}$. Per questo, se a volte il soggetto - come nel passo citato in apertura - sembra "costruire" in maniera determinante il paesaggio e il mondo esterno, altre volte egli sembra completamente in balia degli oggetti circostanti. Saint-Preux, che come Rousseau è caratterizzato da una sensibilità particolarmente sviluppata, sperimenta sino in fondo tale destino: «O Julie, que c'est un fatal présent du ciel qu'une ame sensible! Celui qui l'a reçu doit s'attendre à n'avoir que peine et douleur sur la terre. Vil jouet de l'air et des saisons, le soleil ou les brouillards, l'air couvert ou serein regleront sa destinée, et il sera content ou triste au gré des vents» ${ }^{56}$.

Proprio la coscienza delle contraddizioni personali e, d'altra parte, un'inesausta ricerca dell'unità rappresentano le premesse fondamentali per comprendere il meccanismo dell'immaginazione dello spazio e, conseguentemente, quello della sua organizzazione ${ }^{57}$.

Un primo aspetto che si può considerare come una premessa necessaria per un'analisi più approfondita di tale organizzazione è la compresenza di un livello fisico e di uno morale. Si tratta di una conseguenza logica della delineazione di una morale sensitiva, nella quale si possono ritrovare due elementi ugualmente centrali per l'autorappresentazione rousseauiana: l'esperienza fisica e sensibile e gli slanci affettivi e spirituali. L'importanza di questi due elementi si può riscontrare a pieno nella figura di Julie, colei che più di ogni altro saprà interagire con l'ambiente circostante sino a costruire quel luogo ideale che è Clarens: «Julie a l'ame et le corps également sensibles. La même délicatesse regne dans ses sentimens et dans ses organes $\rangle^{58}$. Per questo motivo l'essere umano «ne peut pas jouir de son existence dans un isolement austère;

(54) À Charles-François-Frédéric de Montmorency-Luxembourg, Maréchal-duc de Luxembourg, Motiers, 20 gennaio 1763 , in J.-J. RousseAu, Correspondance complète de Jean-Jacques Rousseau, edizione critica a cura di R. A. LeIGH, Genève-Oxford, Institut et Musée Voltaire-The Voltaire Foundation, 1965 ss., 51 voll., [2440], vol. XV, janvier-mars 1763, p. 48.

(55) J.-J. Rousseau, Confessions, III, O.C., I, p. 128. Un autoritratto altrettanto suggestivo è delineato nel Persifleur, dove Rousseau si dipinge come un "essere di sensazione", particolarmente mutevole e instabile. Richiamandosi esplicitamente alla quinta Satire di Boileau, egli scrive che «rien n'est si dissemblable à moi que moi-même, c'est pourquoi il seroit inutile de tenter de me définir autrement que par cette varieté singuliére; elle est telle dans mon esprit qu'elle influë de tems à autre jusques sur mes sentimens. [...] C'est cette irrégularité même qui fait le fond de ma constitution». J.-J. RoussEAU, Persifleur, O.C., I, pp. 1108-1109.

(56) J.-J. Rousseau, Julie ou la Nouvelle Héloïse (I, 26), O.C., II, p. 89.

(57) Cfr. R. GRimsley, Rousseau et l'imagination de l'espace, in «Annales Jean-Jacques Rousseau», XXXIX, 1972-1977, pp. 47-64.

(58) J.-J. RoussEAU, Julie ou la Nouvelle Héloïse (V, 2), O.C., II, p. 541. 
il a besoin $[\ldots]$ du monde extérieur» ${ }^{59}$. Così, se da un lato è la ricerca della gioia che porta Rousseau verso il mondo fisico, dall'altro l'elemento materiale e oggettivo rimane imprescindibile per la costruzione di qualsiasi spazio esterno. Infatti, l'animo sensibile e i sentimenti espansivi non possono che estendersi su altri oggetti e la vera gioia non è possibile senza «le concours des objets sensibles» ${ }^{60}$. Questa imprescindibilità del livello fisico vale anche per gli spazi immaginati e non reali, alle cui radici si trova in ogni caso immancabilmente un dato fisico, un oggetto evocatore o un segno rammemorativo (basti richiamare il celebre episodio della pervinca di Madame de Warens) ${ }^{61}$.

In definitiva, si potrebbe formulare nella maniera seguente la regola fondamentale che è alla base della costruzione dello spazio: l'ambiente esterno agisce necessariamente sull'anima, la quale tuttavia lo percepisce secondo le sue emozioni. È evidente come una simile affermazione ponga in evidenza i limiti, che lo stesso Rousseau intuiva perfettamente, di una morale sensitiva intesa in senso meccanicistico: non si potrà mai predisporre l'esterno perché sia sicuramente favorevole alla felicità e alla virtù. Qualsiasi realtà esterna infatti, a partire dalla percezione stessa dello spazio, è interpretata in maniera differente perché si percepisce solo ciò che si sente. Un luogo potrà così condizionare, avviare e favorire il comportamento individuale, pur senza determinarlo. Rousseau, d'altronde, crede troppo nelle potenzialità dell'essere umano per poterlo privare in qualsiasi modo della scelta e della responsabilità (ecco il senso del suo insistere in maniera ossessiva sulla moralità dell'bomme de l'bomme) ed è altresì consapevole che la linea che separa la libertà dal determinismo dipende dalla personalità del singolo.

Fatta salva questa importante premessa, sembra possibile individuare delle regolarità e delle costanti nella delineazione dello spazio esterno ideale. Esso dovrà caratterizzarsi innanzitutto per la sua innocenza e la sua purezza $a^{62}$, qualità che più di tutte le altre consentono all'essere umano il recupero della sua dimensione originaria perduta con l'ingresso nella società civile: si tratta di riscoprire i tratti autentici della statua di Glauco sfigurata dal tempo e dalle intemperie ${ }^{63}$. Da qui scaturisce la preferenza rousseauiana per i paesaggi naturali, il cui fascino sembra l'unico in grado di indirizzare, seppure con eccezioni, verso il bonbeur: «Mais vivifiée par la nature et revetue de sa robe de noces au milieu du cours des eaux et du chant des oiseaux, la terre offre à l'homme dans l'harmonie des trois régnes un spectacle plein de vie, d'intérest et de charme, le seul spectacle au monde dont ses yeux et son cœur ne se lassent jamais ${ }^{64}$. Inoltre, proprio conseguentemente alla ricerca d'innocenza nel mondo esterno, Rousseau «a toujours aimé l'espace clair et pur, surtout lorsqu'il a pu en jouir avec une personne aimée» ${ }^{65}$. Si può considerare esemplificativa, in questo senso, la descrizione della campagna dove si era recato a passeggiare con Madame de Warens: «Tout sembloit conspirer au bonheur de cette journée. Il avoit plu depuis

(59) R. Grimsley, Rousseau et l'imagination de l'espace, cit., p. 50. 816.

(60) J.-J. Rousseau, Dialogues, II, O.C., I, p.

(61) Cfr. J.-J. Rousseau, Confessions, VI, O.C., I, p. 226

(62) Il paesaggio naturale viene a volte indicato esplicitamente come l'unico mezzo in grado di purificare l'animo umano o l'immaginazione, contaminate dagli aspetti nefasti della civilizzazione. Si tratta di una vera e propria "terapia sensualistica": «Brillantes fleurs, email des près, ombrages frais, ruisseaux, bosquets, verdure, venez purifier mon imagination salie par tous ces hideux objets. Mon ame morte à tous les grands mouvemens ne peut plus s'affecter que par des objets sensibles». J.-J. Rousseau, Rêveries, VII, O.C., I, p. 1068.

(63) Cfr. J.-J. Rousseau, Discours sur l'origine de l'inégalité, O.C., III, p. 122. Il riferimento alla statua di Glauco è di ascendenza platonica; cfr. PlatoNE, Repubblica, X, 611 c-d.

(64) J.-J. Rousseau, Rêveries, VII, O.C., I, p. 1062.

(65) R. Grimsley, Rousseau et l'imagination de l'espace, cit., p. 51. 
peu; point de poussiére, et des ruisseaux bien courans. Un petit vent frais agitoit les feuilles, l'air étoit pur, l'horizon sans nuages; la sérénité regnoit au Ciel comme dans nos cœurs $\rangle^{66}$. È evidente, in questo caso, come la persona amata rispecchi a tal punto l'ambiente esterno da fondersi con esso senza alterarne in alcun modo la purezza. Riferendosi proprio a Maman, Jean-Jacques osserva questo fenomeno: «Je faisois de ce charmant paysage encore un des bienfaits de ma chere patronne: il me sembloit qu'elle l'avoit mis là tout exprès pour moi; je m'y plaçois paisiblement auprès d'elle; je la voyois par tout entre les fleurs et la verdure; ses charmes et ceux du printems se confondoient à mes yeux» ${ }^{67}$.

Proprio grazie al sentimento di innocenza originaria che è in grado di trasmettere, il mondo fisico può venire ad assumere una funzione protettrice e riconfortante, che si può definire materna. Riferendosi all'ambiente naturale Rousseau usa spesso l'espressione "mère commune", che sottolinea con efficacia proprio questa caratteristica del luogo ideale:

[La nature est] la mére commune qui se plait à parer le séjour de ses enfans.

[...] alors me réfugiant chez la mére commune j'ai cherché dans ses bras à me soustraire aux atteintes de ses enfans ${ }^{68}$.

La funzione protettrice e materna dello spazio esterno è inoltre ribadita dall'idea che, proprio attraverso il contatto con esso, l'essere umano possa provare la gioia della rinascita. Paradigmatica è in tale senso la descrizione dell'incidente di Ménilmontant $^{69}$, nella quale proprio la percezione del luogo consente il raggiungimento del sentimento dell'esistenza allo stato puro:

La nuit s'avançoit. J'apperçus le ciel, quelques étoiles, et un peu de verdure. Cette prémiére sensation fut un moment délicieux. Je ne me sentois encor que par là. Je naissois dans cet instant à la vie, et il me sembloit que je remplissois de ma legere existence tous les objets que j'appercevois. Tout entier au moment présent je ne me souvenois de rien; je n'avois nulle notion distincte de mon individu, pas la moindre idée de ce qùi venoit de m'arriver; je ne savois ni qui j'étois ni où j'étois; je ne sentois ni mal, ni crainte, ni inquietude. [...] Je sentois dans tout mon être un calme ravissant auquel chaque fois que je me le rappelle je ne trouve rien de comparable dans toute l'activité des plaisirs connus ${ }^{70}$.

Si tratta di un passo di capitale importanza, poiché racchiude alcuni degli elementi fondamentali per comprendere come l'io possa rigenerarsi a contatto con uno spazio esterno riconfortante, aspetto sottolineato ancor di più in questo caso dal senso di disorientamento che segue l'incidente. Innanzitutto, la coscienza dell'io è qui ridotta a una sensazione («Je ne me sentois encor que par là»). Inoltre è molto evidente l'idea di una vera e propria rinascita a contatto con gli oggetti naturali ( Je naissois dans cet instant à la vie»): la coscienza, appena uscita dal nulla accede così all'esse$\mathrm{re}$, ma in maniera ancora infinitamente tenue e imponderabile. Per poter giungere al sentimento dell'esistenza, infatti, il soggetto deve proiettarla sul mondo esteriore («Je remplissois de ma legere existence tous les objets que j’appercevois») che non è

(66) J.-J. Rousseau, Confessions, VI, O.C., I, p. 244.

(67) Ivi, III, O.C., I, p. 105.

(68) J.-J. Rousseau, Dialogues, II; Rêveries, VII, O.C., I, pp. 807 e 1066.

(69) Il 24 ottobre 1776 Rousseau fu fatto cadere da un cane mentre, di ritorno da una passeggiata, scendeva lungo la collina di Ménilmontant. Se la cavò con la slogatura del pollice e del braccio destro e con numerose contusioni. Nel passo qui riportato Jean-Jacques descrive il momento in cui riprende i sensi.

(70) J.-J. Rousseau, Rêveries, II, O.C., I, p. 1005. 
visto in alcun modo come ostile in quanto non ha realtà autonoma; l'io e ciò che lo circonda diventano tutt'uno, a tal punto che il soggetto perde la nozione tanto di se stesso quanto dello spazio («Je ne savois ni qui j’étois ni où j’étois»). Così, rafforzando il sentimento dell'innocenza e della purezza, l'aspetto fisico e sensibile dello spazio materno non permette a coloro che ne entrano in contatto di rimanere in una condizione inattiva o statica. Da qui l'irresistibile richiamo dei luoghi naturali e l'invito a identificarsi o, addirittura, a perdersi in essi, elevandosi poco alla volta al di sopra dei semplici oggetti fisici per smarrirsi nell'immensità del sistema universale: «Je sens des extases, des ravissemens inexprimables à me fondre pour ainsi dire dans le systême des êtres, à m’identifier avec la nature entiére ${ }^{71}$.

L'identificazione con lo spazio, a causa della reciprocità che caratterizza la sua relazione con il soggetto, potrà essere duplice. Se è possibile, come nel caso di Ménilmontant o delle estasi descritte a Malesherbes, una sorta di annientamento dell'io nello spazio, è allo stesso modo possibile che sia il soggetto a vivificare il luogo circostante, trasformandolo e rafforzando contemporaneamente la propria identità. Ronald Grimsley sostiene che proprio questa ultima modalità è quella più propria dell'immaginazione rousseauiana dello spazio: «Malgré ces moments extatiques où Rousseau semble s'oublier complètement, il ne veut pas perdre son identité. Au contraire, il veut pénétrer l'espace de façon à renforcer le sentiment de sa réalité personnelle» ${ }^{72}$. A volte, effettivamente, lo spazio sembra mutare assecondando perfettamente i desideri dell'individuo. Nei momenti di disperazione ad esempio, come nei momenti di grande felicità, anche le cose esterne acquistano un aspetto diverso. Quando SaintPreux è costretto ad allontanarsi dall'amata la sua infelicità pare trasformare qualsiasi oggetto esteriore, sino a deformarne la percezione:

Dans les violens transports qui m'agitent je ne saurois demeurer en place; je cours, je monte avec ardeur, je m'élance sur les rochers; je parcours à grands pas tous les environs, et trouve par tout dans les objets la même horreur qui regne au dedans de moi. On n'apperçoit plus de verdure, l'herbe est jaune et flétrie, les arbres sont dépouillés, le séchard et la froide bise entassent la neige et les glaces, et toute la nature est morte à mes yeux, comme l'espérance au fond de mon cœur $^{73}$.

Più avanti nel romanzo, è lo stesso precettore a teorizzare la possibilità di questa trasformazione: «O Julie! [...] hâtons-nous d'ajouter à ces ornemens du printems la présence de deux amans fidelles: Portons le sentiment du plaisir dans des lieux qui n'en offrent qu'une vaine image; alons animer toute la nature, elle est morte sans les feux de l'amour» ${ }^{74}$.

Questo bisogno di trovarsi in una continua relazione con lo spazio esterno, in una «manière brutale, presque érotique» ${ }^{75}$ - al fine sia di disperdersi nella totalità della natura, sia di sviluppare il sentimento della propria realtà personale - ha delle conseguenze significative. Infatti, nonostante i suoi sogni paradisiaci, Rousseau non

(71) Ivi, VII, O.C., I, pp. 1065-1066.

(72) R. Grimsley, Rousseau et l'imagination de l'espace, cit., p. 54.

(73) J.-J. Rousseau, Julie ou la Nouvelle Héloïse (I, 26), O.C., II, p. 90. Un altro esempio paradigmatico di come la disperazione possa deformare la percezione dello spazio è rappresentato dalla narrazione del vano tentativo (avvenuto il 24 febbraio del 1776) compiuto da Rousseau, ormai sicuro di essere vittima di un complotto universale, di depositare il manoscritto dei Dialogues sull'altare di
Notre-Dame: l'intera cattedrale sembra subire una metamorfosi spaziale così evidente da risultare irriconoscibile e tutti gli oggetti fisici, dalla grata alla porta del coro, sembrano coalizzati contro JeanJacques al fine d'intrappolarlo in una dimensione ostile. Cfr. J.-J. Rousseau, Histoire du précedent ecrit, O.C., I, p. 980.

(74) J.-J. Rousseau, Julie ou la Nouvelle Héloïse (I, 38), O.C., II, pp. 116-117.

(75) R. GRIMSLEY, Rousseau et l'imagination de l'espace, cit., p. 55. 
identificherà mai l'innocenza e la tranquillità con una situazione statica e immutabile. Egli vuole percepire una realtà esterna viva e ricca di sfumature come il mondo interiore: il luogo ideale rousseauiano dovrà essere in grado di soddisfare tutte le esigenze della sua personalità, a partire dalle sensazioni sino a giungere ai sentimenti e, addirittura, all'aspirazione religiosa. Gli spazi si caratterizzeranno così sempre per la loro varietà e la loro ricchezza, non escludendo neppure elementi di contrasto o disordine. D'altronde, come lo stesso Jean-Jacques sottolinea nel nono libro delle Confessions, «le contraste des positions, la richesse et la varieté des sites, la magnificence,

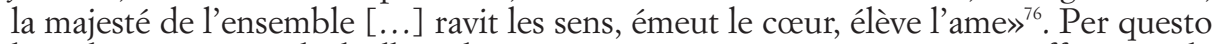
le ombre proiettate dagli alberi, le rocce o i crepacci potranno sortire un effetto simile a quello dei colori vivi e gai dei fiori e delle piante; si tratta tuttavia di suggestioni che potranno svilupparsi solo in quegli animi dotati di una particolare sensibilità: «Ce lieu solitaire - scrive Jean-Jacques riferendosi a Meillerie - formoit un reduit sauvage et desert; mais plein de ces sortes de beautés qui ne plaisent qu'aux ames sensibles et paroissent horribles aux autres» ${ }^{77}$.

Un altro elemento fondamentale che contribuisce a creare la varietà dello spazio è il movimento. Non si tratta però di un moto qualsiasi, ma di quel particolare tipo di movimento che consente all'essere umano di realizzare allo stesso tempo i propri slanci di espansione e delimitazione. Un paesaggio immobile impedisce infatti qualsiasi slancio espansivo al soggetto: egli non può che rimanere assolutamente indifferente dinanzi a una dimensione esterna che non rispecchia alcun elemento della sua varia e mutevole vita interiore. L'assoluta staticità e l'assoluta immobilità non possono che essere associati all'incapacità di provare sensazioni e, in definitiva, alla morte. Tuttavia, anche un paesaggio caratterizzato da un eccessivo movimento impedirà al soggetto di circoscriversi e di rientrare così in se stesso. È lo stesso Rousseau, in una sequenza della quinta promenade (ove lo spazio circoscritto della barca abbandonata al moto lieve delle onde apre innanzi al soggetto orizzonti infiniti), a teorizzare con chiarezza la necessità di questo particolare tipo di movimento che deve caratterizzare lo spazio esterno:

Il n'y faut ni un repos absolu ni trop d'agitation, mais un mouvement uniforme et modéré qui n'ait ni secousses ni intervalles. Sans mouvement la vie n'est qu'une letargie. Si le mouvement est inégal ou trop fort il réveille; en nous rappellant aux objets environnans, il détruit le charme de la rêverie, et nous arrache d'au dedans de nous [...] et nous rendre au sentiment de nos malheurs ${ }^{78}$.

Jean-Jacques non ama dunque lo spazio uniforme, ma cerca piuttosto paesaggi che riflettano le contraddizioni del suo carattere. Solo simili luoghi, infatti, sono in grado di agire sulla sensibilità e sull'immaginazione, che resterebbero inerti dinnanzi ad un'indifferenziata uniformità (ciò a cui si riduceva lo spazio esterno per l'uomo originario). Proprio l'influenza dell'immaginazione è un'altra delle costanti da tener presenti nell'analisi della concezione rousseauiana dello spazio. Gli spazi dell'immaginazione godono infatti di un'importanza sicuramente non inferiore rispetto a quelli della realtà e le due dimensioni finiscono con l'influenzarsi reciprocamente al punto da non risultare sempre distinguibili: non solo l'immaginazione incide profondamente sulla vita reale, ma la realtà si riflette nella dimensione immaginaria, condizionandone la struttura stessa. Continuamente ossessionato dal bisogno di trovare un solido fon-

(76) J.-J. Rousseau, Confessions, IX, O.C., I, p. 431.

(77) J.-J. Rousseau, Julie ou la Nouvelle Héloïse
(IV, 17), O.C., II, p. 518.

(78) J.-J. Rousseau, Rêveries, V, O.C., I, p. 1047. 
damento all'esistenza, Rousseau proverà sempre a coniugare la realtà del mondo fisico con la chimera del dato immaginario, tanto che diventerà spesso impossibile nella sua opera distinguere i luoghi della vita vissuta da quelli sognati. Egli desidera la possibilità di trovare una felicità perfetta, sostenuto in tale speranza dall'intima convinzione nella bontà di Dio e della creazione. Una completa riuscita di tale aspirazione di stabilità è destinata a rimanere preclusa all'essere umano in questa vita, e la contemplazione dell'ordine perfetto sarà la ricompensa degli uomini virtuosi nella vita futura ${ }^{79}$. Al di là di rari e preziosi momenti, riscontrabili ad esempio in alcune rêveries, l'esperienza dello spazio inteso come ordine universale rimane sostanzialmente preclusa all'essere umano. Nella vita terrena - caratterizzata non già da una felicità piena e perfetta, ma da un frêle bonbeur ${ }^{80}$ - bisogna rivolgersi verso l'organizzazione dello spazio fisico circostante.

L'uomo deve dunque riuscire a costruire uno spazio organizzato secondo i propri desideri, in modo tale da garantirsi la stabilità e la pace. Si tratterà, tuttavia, di una "costruzione" e non di una "creazione" vera e propria, perché il dato fisico e naturale rimane comunque imprescindibile. Ciò che è fondamentale è il fatto che l'essere umano «doit avoir la satisfaction de parteciper à la création de son monde, d'y apporter une contribution personnelle» ${ }^{81}$. Questo contributo potrà connotarsi in differenti modi: da un effettivo intervento modificatore (basti pensare alla complessa costruzione della comunità di Clarens o dell'Eliseo di Julie) sino a giungere alla modellazione dello spazio attraverso la sola immaginazione.

Le regolarità sin qui riscontrate in quel procedimento rousseauiano che si può definire "costruzione dello spazio", per sottolineare la reciprocità tra il soggetto e il luogo, rendono consapevoli di come si tratti di un'operazione estremamente complessa e di non facile riuscita: una tale impresa, infatti, richiede il raggiungimento di un delicato equilibrio tra ordine e libertà che non può realizzarsi se lo spazio è troppo grande o troppo vago. È così necessario indagare più da vicino come possa configurarsi tale luogo ideale e, in particolar modo, se sia possibile individuare un'immagine spaziale che lo caratterizzi. Una risposta a tale quesito, o per lo meno un prezioso suggerimento, sembra essere data dallo stesso Rousseau quando esprime il desiderio di possedere «une sphére étroite mais delicieusement choisie, où j'étois assuré de régner» ${ }^{82}$.

\section{L' immagine della sfera}

La sfera, insieme a quella sua proiezione bidimensionale che è il cerchio, è l'unica figura in grado di rappresentare la costruzione del luogo esterno nell'immaginazione di Rousseau, tanto da diventare l'immagine spaziale per eccellenza riscontrabile nelle sue opere.

(79) La concezione rousseauiana del paradiso è incentrata proprio sulle stesse caratteristiche che contraddistinguono il luogo ideale. Lo spazio paradisiaco ha infatti come prerogative «simplicity and innocence» che suggeriscono a loro volta le idee di luce e trasparenza. «For Rousseau paradise always had a translucent quality which made it a worthy abode for God's creatures. Innocence and light are also the source of yet another essential characteristic of paradise-peace. Both innocence and peace are essential to an existence "without division and obstacle"». R. GRIMSLEY, Rousseau and the religious Quest, Oxford, Oxford U. P., 1968, p. 92.

(80) Tale espressione si trova in un celebre passo dell' Émile: «C'est la foiblesse de l'homme qui le rend sociable: ce sont nos miséres communes qui portent nos cœurs à l'humanité, nous ne lui devrions rien si nous n'étions pas hommes. Tout attachement est un signe d'insuffisance: si chacun de nous n'avoit nul besoin des autres il ne songeroit guéres à s'unir à eux. Ainsi de nôtre infirmité même nait nôtre frêle bonheur». J.-J. RoussEAu, Émile, IV, O.C., IV, p. 503. Il concetto di "fragile felicità" è approfondito in un saggio di T. TODOROV, Frêle bonbeur. Essai sur Rousseau, Paris, Hachette, 1985.

(81) R. GRImsley, Rousseau et l'imagination de l'espace, cit., p. 59. 45.

(82) J.-J. Rousseau, Confessions, II, O.C., I, p. 
Questa preferenza accordata alla sfera non dipende semplicemente da una scelta soggettiva e umana, ma si radica a un livello ben più profondo e costitutivo: essa, infatti, è la figura scelta da Dio per creare l'universo e la terra ed è così l'immagine naturale per eccellenza. Tale convinzione emerge con forza in un breve scritto intitolato significativamente Traité de sphère (o Traité élémentaire de sphère). Si tratta di un saggio di astronomia e geografia diviso in undici capitoli, composto secondo la tradizione in una sola mattinata del 1751, sulla scia della probabile influenza del Micromégas di Voltaire ${ }^{83}$. Rousseau descrive dapprima l'evoluzione del sole, le sue conseguenze sulle stagioni e la durata variabile dei giorni, per concentrarsi in seguito sui movimenti terrestri e lunari nell'ambito del sistema copernicano. Infine, egli esamina la sfera terrestre su di un piano prevalentemente idrografico e orografico. Ciò che qui interessa di tale scritto, così inconsueto nel panorama della produzione rousseauiana, non è evidentemente il suo aspetto scientifico, quanto l'idea molto evidente di come la natura sia testimone dell'opera di Dio e di come gli uomini ciechi e malvagi «font du spectacle céleste de ce monde la véritable image de l'Enfer» ${ }^{84}$. Per riconquistare l'originaria dimensione perduta sarà necessario recuperare l'immagine spaziale utilizzata da Dio e dalla natura ${ }^{85}$. La sfera, d'altronde, proprio a causa della sua naturalità, non potrà che ergersi a emblema della stessa esistenza umana: infatti, «chaque homme a ainsi son Cercle particulier ou horizon dont il est le Centre» ${ }^{86}$. L'essere umano e lo spazio circostante, l'interno e l'esterno, possono dunque strutturarsi similmente e ciò garantisce la loro reciprocità e la possibilità di influenzarsi a vicenda. Nella condizione attuale, in cui l'ambiente naturale è stato trasformato dall'uomo nell'immagine dell'inferno, questa possibile consonanza è sostanzialmente perduta e deve essere riconquistata attraverso una riforma (o, per sottolineare di più l'aspetto spaziale, una ricostruzione) di entrambe le dimensioni.

Per quel che riguarda l'interiorità, pare lecito domandarsi innanzitutto che cosa intenda Rousseau quando sostiene che l'essere umano è il centro di una circonferenza. Un'efficace risposta a tale quesito è stata fornita da Georges Poulet, il quale osserva, in uno dei rari saggi dedicati specificamente al valore che l'immagine del cerchio assume nel pensiero di Jean-Jacques: «L'homme est donc un centre, centre de vie expansive. [...] Avant que d'entretenir avec le monde environnant la multiplicité de rapports qui seront les activités de l'âme expansive, ce centre existe déjà, au moins de façon virtuelle» ${ }^{87}$. Senza la possibilità di entrare in contatto con gli oggetti esterni, dunque, le potenzialità umane resterebbero sostanzialmente inespresse. Lo sviluppo di tali sentimenti espansivi è un'operazione particolarmente complessa, poiché è ostacolata dalla debolezza costitutiva dell'individuo: «Nous naissons foibles, nous avons besoin de forces» ${ }^{88}$. Il primo consiglio rivolto alla giovane madre nell'Émile è significativamente quello di costruire intorno al figlio una sfera protettiva e delimitata, in grado di favorirne il corretto sviluppo: «Forme de bonne heure une enceinte autour de l'ame de ton enfant: un

(83) Tale tradizione è riportata da G. STRECKEISEN-MoulTou, che fu nel 1861 il primo editore del Traité de sphère, in Euvres et correspondance inédites de J.-J. Rousseau, pp. 191-212. La genesi dello scritto è in realtà molto più complessa. Cfr. P. SPEZIALI, Textes scientifiques, in O.C., V, p. CCXLVI e ss.; J.-M. DizÈs, voce «Traité de sphère ou Traité élémentaire de sphère», in Dictionnaire de Jean-Jacques Rousseau, cit., pp. 891-892. 601 .

(84) J.-J. Rousseau, Traité de sphére, O.C., V, p.

(85) Nella terza Lettre sur la botanique Rousseau si serve significativamente dell'immagine del cerchio per spiegare a Madame Delessert la configurazione generale dei fiori: «Les fleurs réguliéres [...] sont celles dont toutes les parties s'écartent uniformement du centre de la fleur et aboutiroient ainsi par leur extremité extérieure à la circonférence d'un cercle». J.-J. RousSEAU, Lettres sur la botanique, III, in O.C., IV, p. 1161. 591.

(86) J.-J. Rousseau, Traité de sphére, O.C., V, p.

(87) G. Poulet, Les métamorphoses du cercle, cit., p. 102.

(88) J.-J. Rousseau, Émile, I, O.C., IV, p. 247. 
autre en peut marquer le circuit; mais toi seule y dois poser la barriére» ${ }^{89}$. La superficie iniziale sulla quale può operare l'animo infantile non è così altro che uno spazio esterno esiguo, attorno al quale si eleva una barriera difensiva. Il bambino, d'altronde, è capace di rapportarsi solo con ciò che lo circonda e lo tocca da vicino, a tal punto che il suo animo è più simile $a$ un punto senza dimensione o a un centro senza periferia piuttosto che a una circonferenza vera e propria: «Supposons qu'un enfant eut à sa naissance la stature et la force d'un homme fait, qu'il sortit, pour ainsi dire, tout armé du sein de sa mére comme Pallas du cerveau de Jupiter [...] il n'appercevroit aucun objet hors de lui $[\ldots]$ toutes ses sensations se réuniroient dans un seul point» ${ }^{90}$.

Il bambino, così, ignora ancora sia la natura che le dimensioni dello spazio esterno; non sa se è vuoto o pieno né sino a dove si estende. Tuttavia, sarà proprio il luogo esterno, qualora sia correttamente costruito, a ricoprire un ruolo fondamentale nell'educazione negativa: ci si trova qui dinnanzi a quella parte del processo di apprendimento definita «éducation des choses» ${ }^{91}$, la quale si affianca a quella derivante dagli uomini e dalla natura. Se quest'ultima non dipende in alcun modo dall'essere umano e la seconda dipende esclusivamente da lui, l'educazione delle cose ne dipende solo per alcuni aspetti. Si tratta di un'ulteriore conferma di quella reciprocità che deve sussistere tra l'individuo e lo spazio esterno. Quel centro di energia incomparabile che è il bambino può infatti mettere in atto tutte le sue potenzialità solo grazie a una corretta organizzazione dell'ambiente circostante:

Tout à l'heure nous n'étions occupés que de ce qui nous touche, de ce qui nous entoure immédiatement: tout à coup nous voila parcourant le globe et sautant aux extrémités de l'univers! Cet écart est l'effet du progrès de nos forces et de la pente de nôtre esprit. Dans l'état de foiblesse et d'insuffisance le soin de nous conserver nous concentre au dedans de nous; dans l'état de puissance et de force le desir d'étendre nôtre être nous porte au delà, et nous fait élancer aussi loin qu'il nous est possible ${ }^{92}$.

È evidente come ci sia un contrasto significativo tra l'iniziale concentrazione dell'essere nel fanciullo e la sua vasta dilatazione quando il soggetto incomincia a svilupparsi. Questa constatazione sembrerebbe far emergere, almeno a prima vista, una contraddizione tra quello che si può definire lo slancio eccentrico del soggetto e la necessità di una precisa delimitazione esterna: «Mesurons le rayon de nôtre sphére et restons au centre comme l'insecte au milieau de sa toile, nous nous suffirons toujours à nous-mêmes et nous n'aurons point à nous plaindre de nôtre foiblesse; car nous ne la sentirons jamais» ${ }^{93}$.

Il limite della circonferenza è così altrettanto significativo che il suo centro espansivo. Qualora tale limite non sia sufficientemente delineato o venga oltrepassato, infatti, il soggetto non riuscirà più in alcun modo a soddisfare i propri slanci espansivi e si troverà, esattamente come il fanciullo educato secondo gli erronei accorgimenti tradizionali, «noyé dans une sphere étrangere, lui qui fit si long-tems le centre de la sienne! ${ }^{94}$. La capacità di circoscrivere lo spazio esterno, in modo non egoistico o possessivo, diventa così la peculiarità dell'uomo virtuoso nei confonti di quello malvagio: «La différence est que le bon s'ordonne par raport au tout et que le méchant ordonne le tout par raport à lui. Celui-ci se fait le centre de toutes choses, l'autre mesure son rayon et se tient à la circonférence» ${ }^{95}$.

(89) Ivi, I, O.C., IV, p. 246.

(90) Ivi, I, O.C., IV, p. 280.

(91) Ivi, I, O.C., IV, p. 247.

(92) Ivi, III, O.C., IV, p. 430.
(93) Ivi, II, O.C., IV, p. 305.

(94) Ivi, IV, O.C., IV, p. 514.

(95) Ivi, IV, O.C., IV, p. 602. 
In definitiva, per gioire dei sentimenti espansivi, bisogna necessariamente circoscriverli in uno spazio delimitato: si tratta del nocciolo di quella che Pierre Burgelin ha definito la «dialectique affective» ${ }^{96}$ di Rousseau. Tale dialettica si sviluppa in due tempi: a un primo movimento di circoscrizione e di ripiegamento dell'animo fa seguito un secondo movimento di espansione. Questo secondo momento è una peculiarità imprescindibile del soggetto in quanto «il n'y a pas là un simple besoin psychologique, mais une loi universelle de l'esprit qui révèle ainsi la générosité de sa nature. Dieu lui-même est tel, sa bonté n'est autre qu'une expansion créatrice» ${ }^{97}$. Tuttavia non ci potrà essere alcuna espansione senza una precedente delimitazione, alcuna aspirazione all'univers senza la retraite, com'è confermato dalla nota battuta dell'ultima promenade: «J'ai besoin de me recueillir pour aimer» ${ }^{98}$.

Si può così comprendere più a fondo l'insistenza sistematica sulla limitatezza spaziale della sfera umana, unica caratteristica in grado di consentire allo stesso tempo il contatto con l'esterno e la protezione dallo stesso:

Ma modération m'inscrivoit dans une sphére étroite mais delicieusement choisie, où j'étois assuré de régner"9.

[...] j'étois déterminé $[\ldots]$ à me renfermer pour le reste de mes jours dans la sphére étroite et paisible pour laquelle je me sentois né ${ }^{100}$.

N'attends pas de moi de longs preceptes de morale, je n'en ai qu'un seul à te donner, et celui-là comprend tous les autres. Sois homme; retire ton cœur dans les bornes de ta condition. Etudie et connois ces bornes; quelque étroites qu'elles soient, on n'est point malheureux tant qu'on s'y renferme ${ }^{101}$.

Dunque, qualora l'essere umano riesca a raggiungere quel prezioso equilibrio tra l'espansione dell'interiorità (il centro del cerchio) e la delimitazione dell'esteriorità (la circonferenza vera e propria), potrà ritrovare nella pienezza del mondo esteriore quella del suo essere intimo. Questi due tipi di pienezza saranno della stessa specie perché l'interiorità e l'esteriorità saranno costruite analogamente e potranno entrare in contatto tra loro sino a fondersi: l'essere intimo, da un lato, potrà espandersi nello spazio esterno e sarà riempito da esso, mentre quest'ultimo sarà delimitato dall'interiorità, la quale diviene la misura del suo sviluppo. Tale duplice movimento si manifesta in diversi passaggi dei primi libri delle Confessions e in particolar modo quando il giovane Jean-Jacques, attraversando per la prima volta le Alpi, pare rendersi conto di come la bellezza del mondo e quella del suo essere intimo siano inscindibili:

Nul accident ne troubla mon voyage; j'étois dans la plus heureuse situation de corps et d'esprit où j'aye été de mes jours. Jeune, vigoureux, plein de santé, de sécurité, de confiance en moi et aux autres, j'étois dans ce court mais précieux moment de la vie où sa plénitude expansive étend pour ainsi dire notre être par toutes nos sensations, et embellit à nos yeux la nature entiére du charme de notre existence ${ }^{102}$.

In questo, come in molti altri casi, si può assistere alla fusione dell'interiorità con lo spazio esterno: il soggetto, infatti, non percepisce semplicemente delle cose, ma il suo modo di percepire le cose e il sentire diventa inevitabilmente un sentirsi. Dal centro dell'io sino all'ultimo punto del paesaggio abbracciato con lo sguardo non vi è più alcuna differenza di natura. Solo in ciò può consistere la vera felicità:

(96) P. Burgelin, op. cit., p. 151.

(97) Ibidem.

(98) J.-J. Rousseau, Rêveries, X, O.C., I, p. 1099.

(99) J.-J. Rousseau, Confessions, II, O.C., I, p. 45.
(100) Ivi, X, O.C., I, p. 515.

(101) J.-J. Rousseau, Émile, V, O.C., IV, p. 819. 57-58. 
«être à la circonférence ce que l'on est au centre» ${ }^{103}$. In una simile situazione, che è sempre caratterizzata dalla compresenza di delimitazione e espansione, l'universo intero non si estende più lontano dello sguardo umano ed è interamente abbracciato dalla conoscenza sensibile. In questo caso non è più possibile tracciare alcuna distinzione tra lo spazio dell'interiorità e quello esterno ed è per questo che sembra lecito parlare di "pienezza espansiva" nonostante l'elemento della delimitazione e quello della finitezza siano sempre presenti. Lo spazio esterno diventa un prolungamento di quello spazio interiore e soggettivo in cui regna la forza espansiva dell'anima che assorbe tutto ciò che sente e lo trasforma nel suo essere proprio. La stessa coscienza, parallelamente, non potrebbe in alcun modo esprimere le sue potenzialità espansive se non si lasciasse assorbire dagli oggetti esterni sino, a tratti, a svanire in loro. Questo duplice movimento, per certi aspetti sconcertante ma mai contraddittorio, è descritto con penetrazione nelle prime righe dell'ottava promenade:

Quand tout étoit dans l'ordre autour de moi, quand j'étois content de tout ce qui m'entouroit et de la sphére dans laquelle j'avois à vivre je la remplissois de mes affections. Mon ame expansive s'étendoit sur d'autres objets, et sans cesse attiré hors de moi par des gouts de mille espéces, par des attachemens aimables qui sans cesse occupoient mon cœur je m'oubliois en quelque façon moi-même, j'étois tout entier à ce qui m'étoit étranger ${ }^{104}$.

Attraverso l'identificazione del più intimo io con l'intero universo di cui è parte, l'essere umano può così trascendere la propria condizione e dilatare la propria esistenza dal limitato spazio terrestre all'intera sfera dell'universo: «L'espace n'est pas ta mesure, l'univers entier n'est pas assés grand pour toi; tes sentimens, tes desirs, ton inquiétude, ton orgueil même, ont un autre principe que ce corps étroit dans le quel tu te sens enchaîné» ${ }^{105}$. Questo sentimento trova la sua più efficace formulazione nella magnifica iperbole "j'etouffois dans l'univers" utilizzata in una delle lettere a Malesherbes $^{106}$.

La dialettica tra espansione e delimitazione trova così la sua risoluzione nell'identità estatica tra la sfera interiore dell'anima e la sfera esteriore dell'universo ${ }^{107}$. Non si tratta di un superamento di uno dei due estremi, quanto del raggiungimento di un perfetto equilibrio tra di loro:

En un sens, je suis une force remplissant tout, dilatée partout; en un autre, ce sont les objets de l'expérience sensible qui, aspirant en eux cette force, s'en servent pour remplir mon esprit de leurs caractéristiques propres. De sorte que tantôt le monde me semble faire partie intégrante de moi-même, et tantôt je crois faire partie intégrante du monde et m'identifier tout à tour avec les mille objets qu'il contient et qui se font percevoir en moi $^{108}$.

(103) G. Poulet, Les métamorphoses du cercle, cit., p. 105.

(104) J.-J. RoussEau, Rẹveries, II, O.C., I, p. 1074.

(105) J.J. Rousseau, Emile, IV, O.C., IV, p. 585.

(106) «J'aimois à me perdre en imagination dans l'espace, mon cœur resserré dans les bornes des etres s'y trouvoit trop à l'etroit, j'etouffois dans l'univers, j'aurois voulu m'elancer dans l'infini». J.J. Rousseau, Lettre à Malesherbes, 26 janvier 1762, O.C., I, p. 1141.

(107) Il concetto di unione estatica è fondamentale nella costruzione dello spazio ideale rousseauiano. All'interno del quadro naturale è possibile distinguere tra due tipi fondamentali di estasi, che in realtà si pongono su di un livello di continuità: (1) il semplice bonbeur derivante dai sensi; (2) l'estasi cosmica, derivante dalla compenetrazione con il sistema degli esseri. La concezione dell'estasi rousseauiana è così molto distante da quella tradizionale dei mistici, in quanto si basa su due poli che in quest'ultima sono assenti, cioè l'importanza del luogo esteriore e della pienezza interiore. A tale proposito Marcel Raymond parla di mystyque naturelle. Cfr. M. Raymond, Jean-Jacques Rousseau. Deux aspects de sa vie intérieure, in «Annales Jean-Jacques Rousseau», XXIX, 1941-1942, pp. 7-57.

(108) G. Poulet, Les métamorphoses du cercle, cit., p. 107. 
Sino a ora si è mostrato come l'immagine della sfera sia in grado di rappresentare spazialmente le esigenze di espansione e delimitazione del singolo individuo, tuttavia la stessa immagine finisce con il diventare il simbolo anche della vita associata. Rousseau, infatti, ha avuto l'indubbio merito di sollevare sempre i problemi da lui trattati al di sopra dell'individuo per rivolgerli anche verso la società. Passando dal piano dell'io a quello del noi, ci si rende conto di come la costruzione della spazio societario ideale non possa che riproporre le stesse caratteristiche di quello individuale: anche in questo caso, difatti, l'obiettivo a cui tendere è una comunità che si potrebbe definire "sferica", poiché ristretta e ben delimitata da un lato, ma in grado al contempo di garantire la massima libertà a tutti i suoi membri. In tale situazione, i centri espansivi dei singoli individui finirebbero con convergere e coincidere con il moi commun e con la volontà generale, vero e proprio centro motorio della sfera statale.

Questa prospettiva giustifica l'estrema importanza attribuita, nella delineazione delle forme di governo, alla dimensione degli Stati e al numero dei cittadini. In particolar modo il governo democratico esigerebbe come condizioni di realizzabilità uno Stato piccolissimo in cui tutti si conoscessero e fossero pressoché pari in ricchezza ed estrazione sociale; si verrebbe così a creare una comunità sobria e virtuosa, una vera e propria sfera a misura uomo. Proprio a causa di questa sua perfezione, la democrazia resterà sostanzialmente un ideale irraggiungibile per degli esseri imperfetti come gli uomini: «S'il y avoit un peuple de Dieux, il se gouverneroit Démocratiquement. Un Gouvernement si parfait ne convient pas à des hommes» ${ }^{109}$. Se è vero che storicamente il governo democratico ha mostrato, come nell'esempio classico di Atene, una tendenza invincibile alle guerre civili e a una perpetua instabilità, è altrettanto vero che Jean-Jacques si rifiuterà sempre di relegare il proprio progetto politico nel regno dell'utopia. Egli non cesserà mai infatti di cercare «une nation libre, paisible et sage qui n'a ni peur ni besoin de personne, qui se suffit à elle-même et qui est heureuse» ${ }^{10}$.

In questa direzione, per altro, sono indirizzate anche tutte le opere in cui Rousseau adatta i principi del diritto politico da lui formulati alle concrete esigenze di una realtà sociale ben determinata, come nei casi della Corsica rivoluzionaria di Pasquale Paoli e della Polonia "anarchica" di Augusto Stanislao Poniatowski. Egli delinea sempre Stati indipendenti e sostanzialmente separati dal resto del mondo (la Corsica si può avvantaggiare del fatto di essere un'isola, mentre per la Polonia viene accentuata maggiormente una separatezza morale più che geografica), caratterizzati da un'autarchia tanto morale quanto economica, nonché dalla più assoluta unanimità di intenti dei cittadini. Solo in questo modo è possibile creare una société bien ordonnée 111 , cioè un luogo "sferico" e a misura d'uomo nel quale è possibile vivere nella trasparenza senza bisogno di alcuna pericolosa mediazione:

Grandeur des Nations! Etendue des Etats! prémiére et principale source des malheurs du genre humain, et surtout des calamités sans nombre qui minent et détruisent les peuples policés. Presque tous les petits Etats, républiques et monarchies indifferemment, prospèrent par cela seul qu'ils sont petits, que tous les citoyens s'y connoissent mutuellement et s'entreregardent, que le chefs peuvent voir par eux-mêmes le mal qui se fait, le bien qu'ils ont à faire; et que leurs ordres s'exécutent sous leurs yeux ${ }^{112}$.

(109) J.-J. Rousseau, Du contrat social (III, 5), O.C., III, p. 406.

(110) J.-J. Rousseau, Considérations sur le gouvernement de Pologne, XI, O.C., III, p. 1003.

(111) Cfr. M. VIroli, Jean-Jacques Rousseau and the "well-ordered society", Cambridge, Cambridge U.P., 1988 (ed. italiana Bologna, il Mulino, 1993).

(112) J.-J. Rousseau, Considérations sur le gouvernement de Pologne, O.C., III, p. 970. 
La costruzione di una società circoscritta diventa così una delle più importanti aspirazioni rousseauiane, aspirazione destinata a trovare numerose e celebri concretizzazioni, come Clarens nella Nouvelle Hélö̈se o la comunità dei Montagnons nella Lettre à d'Alembert sur les spectacles ${ }^{113}$. Sin dalla prefazione dialogata del suo romanzo filosofico Rousseau mette significativamente in luce come la vicenda sia ambientata «dans la petite sphere de deux ou trois Amants ou Amis toujours occupés d'eux seuls» ${ }^{114}$. Solo all'interno di tale petite sphère «on apprend à aimer l'humanité», mentre «dans les grandes sociétés on n'apprend qu'à haïr les hommes» ${ }^{115}$. Clarens è indubbiamente una sfera separata, nella quale regna la più assoluta trasparenza; non vi è infatti alcuna differenza tra le "anime belle" che abitano tale luogo e lo spazio circostante. Ognuno, così, può sviluppare pienamente i propri sentimenti espansivi e, grazie all'assoluta fiducia che apre l'una all'altra le anime, può riconquistare i privilegi dell'innocenza e della purezza:

Convenez, du moins, que tout le charme de la société qui régnoit entre nous est dans cette ouverture de cœur qui met en commun tous les sentimens, toutes les pensées, et qui fait que chacun se sentant tel qu'il doit être se montre à tous tel qu'il est. Supposez un moment quelque intrigue secrette, quelque liaison qu'il faille cacher, quelque raison de reserve et de mistere; à l'instant tout le plaisir de se voir s'évanouït, on est contraint l'un devant l'autre, on cherche à se dérober, quand on se rassemble on voudroit se fuir ${ }^{116}$.

Ci si trova di fronte a un mondo di unanimità dove, esattamente come nella società del Contrat social, nessuna volontà particolare può isolarsi dalla volontà generale. Nel caso di Clarens, inoltre, la piccola comunità circoscritta ha il suo centro in Julie, la cui anima si comunica a tutti coloro che la circondano rendendo possibile quella "comunicazione ristretta" che sarà una caratteristica costante di ogni spazio comunitario costruito secondo l'immagine della sfera. La sintonia interna in una «société très-intime» sarà così forte che «les amis confondant leurs ames, confondent aussi leurs manieres de penser, de sentir, et de dire. Cette Julie, telle qu'elle est, doit être une créature enchanteresse; tout ce qui l'approche doit lui ressembler; tout doit devenir Julie autour d'elle» ${ }^{117}$. Julie, d'altronde, appartiene a quelle «ames d'une certaine trempe» che «transforment pour ainsi dire les autres en elles-mêmes; elles ont une sphere d'activité dans laquelle rien ne leur resiste ${ }^{118}$. Questa sorta d'irraggiamento che parte dalla protagonista del romanzo costituisce il vero principio morale che rende possibile la comunicazione tra gli animi. La stessa Julie pare consapevole di come, nel suo mondo, non vi sia alcuna differenza tra se stessa, la cerchia dei suoi amici e lo spazio esterno:

[...] je suis environnée de tout ce qui m'intéresse, tout l'univers est ici pour moi; je jouïs à la fois de l'attachement que j'ai pour mes amis, de celui qu'ils me rendent, de celui qu'ils ont l'un pour l'autre; leur bienveuillance mutuelle ou vient de moi ou s'y rapporte; je ne vois rien qui n'étende mon être, et rien qui le divise; il est dans tout ce qui m'environne, il n'en reste aucune portion loin de moi $^{119}$.

(113) La comunità dei Montagnons è significativamente rappresentata come un mondo, caratterizzato da una separatezza sia geografica che morale, costituito da tante sfere che trovano il proprio centro nelle singole abitazioni, garantendo un perfetto equilibrio tra la retraite e l'univers: «Je me souviens d'avoir vu dans ma jeunesse aux environs de Neufchâtel un spectacle assés agreable et peut être unique sur la terre. Une montagne entiére couverte d'habitations dont chacune fait le centre des terres qui en dépendent; en sorte que ces maisons, à distances aussi égales que les fortunes des proprie- taires offrent à la fois aux nombreux habitans de cette montagne le recueillement de la retraite et les douceurs de la Societé». J.-J. RousseAu, Lettre à $M$. d'Alembert, O.C., V, p. 55.

(114) J.-J. RoussEAu, Julie ou la Nouvelle Héloüse, Préface, O.C., II, p. 14.

(115) Ibidem.

(116) Ivi (VI, 8), O.C., II, p. 689.

(117) Ivi, Préface, O.C., II, p. 28.

(118) Ivi (II, 5), O.C., II, p. 204.

(119) Ivi (VI, 8), O.C., II, p. 689. 
Alla fanciulla è così riuscito l'arduo compito di bilanciare le esigenze umane di espansione e delimitazione, sino a creare quella che Grimsley ha definito efficacemente «une société circonscrite mais assez large pour être humaine» ${ }^{120}$.

Questi brevi accenni al microcosmo di Clarens consentono inoltre di mostrare come l'immagine della sfera sia alla base tanto dell'organizzazione dello spazio reale quanto di quello immaginario. Le leggi che regolano il mondo sognato da Rousseau, evocato quasi sempre con il nome di "paese delle chimere" ${ }^{121}$, rispecchiano d'altronde a loro volta i movimenti della dialettica affettiva interiore. Se l'immaginazione, che si caratterizza sempre come una facoltà creatrice che agisce in absentia rispetto all'oggetto immaginato, disperde e dilata l'anima umana, quest'ultima tuttavia trova un momento di forte delimitazione nella memoria. L'immaginazione e la memoria, le due facoltà alla base del mondo fittizio, finiscono così per delimitarlo a sua volta in una sfera circoscritta, l'unica in grado di rivelare all'uomo il suo essere più essenziale: «Le monde réel a ses bornes, le monde imaginaire est infini; ne pouvant élargir l'un retrécissons l'autre; car c'est de leur seule différence que naissent toutes les peines qui nous rendent vraiment malheureux» ${ }^{122}$.

In definitiva, volendo sintetizzare le caratteristiche proprie spazio sferico rousseauiano, che coincidono con le peculiarità di tutti i luoghi ideali rintracciabili nella sua opera, ci si rende conto di come la prima e fondamentale connotazione della sfera sia la sua naturalezza. Si tratta infatti non solo della figura scelta da Dio per creare l'universo e la terra, ma anche dell'immagine in grado di rappresentare l'esistenza umana, descritta come un cerchio particolare di cui l'individuo è il centro. Alla dimensione naturale e originaria della circonferenza e della linea curva si oppone la dimensione artificiale della linea retta, emblema della violenza culturale umana. Una significativa conferma di ciò si ritrova nell'affermazione della Nouvelle Héloïse secondo cui «la nature ne plante rien au cordeau» ${ }^{123}$.

Proprio la naturalezza della sfera ne fa l'unica immagine in grado di rappresentare al contempo lo spazio esteriore e quello interiore: questa analoga struttura rende possibile la reciprocità tra le due dimensioni. L'animo umano si può infatti paragonare al centro espansivo della sfera; esso, tuttavia, finirebbe con il disperdersi completamente ${ }^{124}$ se non trovasse dei limiti ben precisi nella circonferenza esterna.

(120) R. GRIMSLEy, Rousseau et l'imagination de l'espace, cit., p. 61.

(121) Per bocca di Julie Rousseau teorizza apertamente la superiorità del paese delle chimere rispetto al mondo reale: «Le pays des chimeres est en ce monde le seul digne d'être habité, et tel est le néant des choses humaines, qu'hors l'Etre existant par lui-même, il n'y a rien de beau que ce qui n'est pas». J.-J. RousseAu, Julie ou la Nouvelle Héloïse (VI, 8), O.C., II, p. 693. E interessante osservare come la stessa idea di paese, contrapponendosi a quella di chimera, rinvii a una dimensione spaziale effettiva, a un mondo autosufficiente che esiste e in cui si può vivere. A tale proposito cfr. L. SozzI, Il paese delle chimere. Aspetti e momenti dell'idea d'illusione nella cultura occidentale, Palermo, Sellerio, 2007, in particolar modo pp. 135-182; P. Sosso, Jean-Jacques Rousseau. Imagination, illusions, chimères, prefazione di L. Sozzi, Paris, Champion, 1999.

(122) J.-J. Rousseau, Émile, II, O.C., IV, p. 305.

(123) J.-J. Rousseau, Julie ou la Nouvelle Héloïse (IV, 11), O.C., II, p. 479. Nel giardino di Julie, al contrario, non c'è alcuna violenza culturale. In esso non c'è nulla di esotico, di strano o di estraneo alla natura stessa e vi ritrova unicamente solo ciò che vi si sarebbe potuto trovare anche spontaneamente, se si fossero cioè prodotte tutte quelle condizioni favorevoli che solo l'intervento umano ha saputo e potuto riunire: «Il est vrai, dit-elle, que la nature a tout fait, mais sous ma direction». Ivi, O.C., II, p. 472 .

(124) Questo pericolo è descritto con penetrazione nella sesta Lettre à Sophie, ove viene utilizzata nuovamente - seppure con una valenza negativa - la metafora del ragno e della tela, già incontrata nell'Émile: «Quand je vois chacun de nous sans cesse occupé de l'opinion pubblique étendre pour ainsi dire son existence tout autour de lui sans en reserver presque rien dans son propre cœur, je crois voir un petit insecte former de sa substance une grande toile par laquelle seule il paroit sensible tandis qu'on le croiroit mort dans son trou. La vanité de l'homme est la toile d'araignée qu'il tend sur tout ce qui l'environne. L'une est aussi solide que l'autre, le moindre fil qu'on touche met l'insecte en mouvement». J.-J. RouSSEAU, Lettres morales, VI, O.C., IV, p. 1112. 
Senza questo duplice movimento l'individuo non potrebbe mai giungere pienamente né al contatto con lo spazio esterno né a una efficace protezione dallo stesso. Tale riflessione fa emergere la connotazione eminentemente protettiva della sfera, caratteristica che, come si è constatato precedentemente, è uno dei requisiti fondamentali ricercati da Rousseau nel processo della costruzione spaziale.

Nel luogo ideale, inoltre, non vi sarà più alcuna differenza tra il centro della sfera, cioè l'espansione dell'interiorità, e la sua circonferenza, cioè la delimitazione dell'esteriorità, ma una assoluta fusione: lo spazio sferico verrà così a caratterizzarsi per la sua uniformità. L'omogeneità e l'uniformità in questione devono essere sempre riferite all'analoga costruzione della dimensione interna ed esterna e non al semplice oggetto circostante. Non vi è dunque alcuna contraddizione tra la preferenza rousseauiana per paesaggi contrastati e non uniformi e l'idea che questi ultimi possano rispecchiare in modo omogeneo le contraddizioni del suo carattere.

Proprio l'omogeneità della sfera ne garantisce l'assoluta trasparenza al suo interno. Qui l'animo umano non incontrerà alcun ostacolo e questo renderà possibile una vera e propria rigenerazione dell'individuo, che lo potrà condurre al raggiungimento del sentimento dell'esistenza allo stato puro o, addirittura, a quello della rinascita.

Tutte le qualità sin qui ricordate contribuiscono a caratterizzare l'immagine della sfera come essenzialmente separata. Si tratta di una separatezza che è in primo luogo spaziale, ma che diventa inevitabilmente anche temporale. A partire dallo spazio, infatti, pare possibile sconfiggere l'inesorabile fluire del tempo: solo nella protettiva circonferenza del luogo ideale l'individuo può contemporaneamente estendersi infinitamente nello spazio, grazie a una sorta di fusione estatica con la natura, e raggiungere la dimensione tanto agognata e assolutamente atemporale dell'autentica felicità: «Le bonheur que mon cœur regrette n'est point composé d'instans fugitifs mais un état simple et permanent» ${ }^{125}$.

L'immagine della sfera, proprio a causa della sua assoluta separatezza, è caratterizzata in ultima istanza da una lacerante dialettica del fuori e del dentro. Si tratta di una dialettica che possiede quell'affilata nettezza che Rousseau riconobbe sempre come propria: «En fait de bonheur et de jouissance, il me falloit tout ou rien» ${ }^{126}$. All'interno della sfera, che rappresenta la naturalezza, la protezione e la trasparenza si oppone tutto ciò che da essa è escluso, cioè lo spazio dell'artificio, pericoloso e opaco. Ciò che viene escluso dalla costruzione di quella circonferenza che è il luogo ideale rousseauiano non è infatti meno significativo di ciò che vi viene incluso: uno sfondo di tenebre è sempre necessario per far risaltare la luminosa gioia dell'innocente mondo di Jean-Jacques, gioia che egli potrà gustare sino in fondo proprio perché ha sperimentato la disperazione di vivere in un mondo ostile.

Ma nonostante tutte le difficoltà derivanti dall'esterno o insite nella fragilità che contraddistingue la condizione umana, in Rousseau non verrà mai meno la fiducia che gli individui possano ritrovare, nella rassicurante dimensione della sphère étroite, una libertà e una felicità degne dell'uomo.

MARCO MENIN

(125) J.-J. Rousseau, Rêveries, V, O.C., I, p. 1046.

(126) J.-J. Rousseau, Confessions, IX, O.C., I, p. 422. Nel secondo Dialogues Rousseau sostiene che
Jean-Jacques non è in grado di animarsi con moderazione: «Il faut qu'il soit de flamme ou de glace; quand il est tiede, il est nul». O.C., I, p. 804. 